Research Article

\title{
Hydrodynamic Analysis of Partially Filled Liquid Tanks Subject to 3D Vehicular Manoeuvring
}

\author{
Mengmeng Han $\left(\mathbb{D},{ }^{1}\right.$ Jian Dai $(\mathbb{D})^{2}$ C. M. Wang $\mathbb{D}^{1},{ }^{1}$ and K. K. Ang $\mathbb{D i}^{3}$ \\ ${ }^{1}$ School of Civil Engineering, The University of Queensland, St Lucia, Queensland 4072, Australia \\ ${ }^{2}$ Department of Marine Technology, Norwegian University of Science and Technology, 7491 Trondheim, Norway \\ ${ }^{3}$ Department of Civil and Environmental Engineering, National University of Singapore, Singapore 117576
}

Correspondence should be addressed to Jian Dai; jian.dai@ntnu.no and C. M. Wang; cm.wang@uq.edu.au

Received 16 April 2019; Revised 8 July 2019; Accepted 28 August 2019; Published 27 November 2019

Academic Editor: Chao Tao

Copyright (c) 2019 Mengmeng Han et al. This is an open access article distributed under the Creative Commons Attribution License, which permits unrestricted use, distribution, and reproduction in any medium, provided the original work is properly cited.

\begin{abstract}
This paper is concerned with liquid sloshing in a partially filled container due to 3-dimensional vehicle motion. The liquid sloshing is described by a set of linear modal equations derived from the potential flow theory, which can be applied to liquid sloshing induced by arbitrary combination of lateral, longitudinal, and rotational excitations. The sloshing force and moment are expressed with a set of hydrodynamic coefficients that are determined by the linear velocity potential. These coefficients can be precalculated and incorporated into the motion equations of the vehicle system so that a fully coupled vehicle-sloshing model is available. In addition, we propose an approach to calculate the hydrodynamic coefficients using the outputs of commercial frequency-domain boundary element software in order to maximize the efficiency of modelling and computation. The accuracy of the proposed model is examined by comparison with available CFD and model test data in the literature. The case of a road tanker encountering a road bump during acceleration/braking is investigated. Results show that the tank rotational motion will affect the amplitude and the sloshing force, and neglecting tank rotation may lead to underestimation of the sloshing force magnitude.
\end{abstract}

\section{Introduction}

Liquid sloshing frequently takes place when vehicles carrying partially filled tanks are subject to acceleration and has attracted researchers' interest in the fields of aerospace, marine, road, and rail engineering. Sloshing in zero-gravity environment has been investigated for space vehicles with partially filled fuel tanks [1]. In marine vessels such as oil and gas tankers, the sloshing induced by ocean waves and its coupling with the ship motion have been extensively investigated [2-4]. In road/rail vehicles, the most well-known circumstance is the sloshing induced by the accelerating, braking, and turning manoeuvring of vehicles. Abrupt vehicle acceleration would cause transient sloshing in the tank, which would last for a period of time even after the external excitation vanishes. Previous studies $[5,6]$ have shown that liquid sloshing is often an undesirable phenomenon which may increase the risk of rollover and reduce the manoeuvrability of the vehicle. In addition, sloshing exerts extra force and moment on the vehicle as well, which affects the running safety and the structural integrity of the vehicle. In practice, baffles are often installed in liquid tanks to reduce the sloshing effect [6-10].

Sloshing has been studied by numerical methods such as the finite element method (FEM) [11], the computational fluid dynamics (CFD) method $[3,12]$, or the smoothed particle hydrodynamics (SPH) method $[13,14]$. For most practical problems, the sloshing is determined by both the tank properties and the motion of the vehicle carrying it. As a result, coupled vehicle-sloshing analysis often requires iterative interaction between two different solvers. For example, the coupled SPH-FEM analysis in [2], coupled BEMCFD in [4], and BEM-FEM [10, 15] studies in offshore engineering have been carried out. Although effective in solving violent coupled vehicle-sloshing problems, the computational effort is intensive. On the other hand, it has been observed that if the sloshing is relatively mild, the free surface does not break and the sloshing motion is not chaotic 
[16], and its motion pattern is analogous to a mechanical system. Therefore, many past studies simplify the sloshing liquid into a spring-mass $[17,18]$ or a pendulum $[19,20]$ system. The complication encountered in this approach is that since the mechanical analogy is not based on the fluid theory, it is hard to derive the parameters (mass, stiffness, damping, and natural frequency) of the analogical model directly, unless the tank geometry is simple such as a rectangular tank. A CFD analysis or a model test has to be carried out in advance to calibrate the parameters of the mechanical model. In this way, the expensive computational cost of CFD simulations still cannot be avoided although a fully coupled mechanical liquid-vehicle model could be established. In addition, most of the current studies based on mechanical models focus only on sloshing excited by translational accelerations, while in reality vehicle rotation will also take place when travelling on uneven road surface, such as a road bump. Unfortunately, studies accounting for the effect of vehicle rotational motion on liquid sloshing motion are rare.

For an incompressible, inviscid, and irrotational fluid, sloshing motion satisfies the Laplace equation and can be described by a velocity potential. Based on the linear potential flow theory, the simplified fluid motion can be described by a set of linear modal equations in a similar form as the motion equation of a mechanical analogy model. In 1998, Bogomaz et al. [21] proposed that the liquid sloshing be considered as a set of pendulums whose mass and stiffness properties are related to the hydrodynamic coefficients that are dependent on the tank geometry and fluid density. Since simple analytical solution to these coefficients may not exist for random tank shapes other than simple geometries such as rectangular or vertically placed cylindrical tanks, it is highly desirable that a computationally efficient numerical approach still needs to be established for evaluating the hydrodynamic coefficients. For example, both linear and nonlinear boundary element methods $[8,22]$ have been employed to evaluate the hydrodynamic coefficients by solving the velocity potential. Semianalytical methods to get the velocity potential and eigenvalues for certain tank geometry have also been investigated [23]. A lot of commercial programs are able to solve the linear sloshing problem induced by harmonic external excitations, but for transient sloshing, extra effort is needed for the frequency domain solution due to the frequency-dependent properties.

This paper studies the sloshing problem inside a road tanker that is subject to an abrupt acceleration. The 6-DOF liquid motion is described by modal equations based on linear potential flow theory. A commercial BEM solver is employed to evaluate the hydrodynamic coefficients for modelling the sloshing motion. The accuracy of the proposed approach has been examined by comparing the results with available CFD simulations and model test results that are available in the open literature. A parametric study has also been carried out to investigate the liquid sloshing under combined 3-dimensional vehicle translational and rotational prescribed accelerations. In particular, the effect of vehicle pitch motion due to a road bump or a road pit on the liquid motion and sloshing force is examined.

\section{Liquid Sloshing inside Container}

Consider a rigid container partially filled with liquid, with the Cartesian coordinates system shown in Figure 1. According to the linear potential flow theory, the fluid is assumed to be incompressible, inviscid, and irrotational. Under the assumptions, the velocity field of the fluid in the entire fluid domain can be described by a velocity potential $\phi$ which satisfies the continuity equation, i.e.,

$$
\frac{\partial^{2} \phi}{\partial x^{2}}+\frac{\partial^{2} \phi}{\partial y^{2}}+\frac{\partial^{2} \phi}{\partial z^{2}}=0
$$

On the free surface $y=\eta$, the kinematic (2) and dynamic free surface boundary conditions have to be satisfied:

$$
\begin{array}{r}
\frac{\partial \phi}{\partial n}-\nabla \eta \cdot \nabla \phi=\frac{\partial \eta}{\partial t} \\
\frac{\partial \phi}{\partial t}+g \eta+\frac{1}{2}(\nabla \phi)^{2}=0 .
\end{array}
$$

The above boundary conditions are nonlinear. Higher order terms come from the convective derivatives in equation (2) and the kinetic energy term in equation (3). For linear sloshing problems, the free surface elevation is considered to be small. Thus, the higher order terms in the above equations are neglected. The boundary conditions are linearized as below at the mean water level as $\eta \longrightarrow 0$ :

$$
\begin{aligned}
\frac{\partial \phi}{\partial y} & =\frac{\partial \eta}{\partial t} \text { on MWL, } \\
\frac{\partial \phi}{\partial t}+g \eta & =0 \text { on MWL. }
\end{aligned}
$$

Note that the assumption that the free surface elevation is small is appropriate for gentle and mild sloshing discussed in this paper. When violent sloshing takes place, the contribution of the higher order terms should not be neglected. Further, the solid-wall boundary condition is satisfied on the rigid tank wall:

$$
\frac{\partial \phi}{\partial n}=v_{n} \text { on } S_{\mathrm{B}},
$$

where $\eta$ is the free surface elevation and $v_{n}$ is the velocity of rigid body motion on body surface $S_{\mathrm{B}}$. In addition, since the fluid domain in sloshing problem is a confined space and the free surface is non-breaking in potential flow problems, the fluid in tank should satisfy overall fluid volume conservation, which requires the integral of free surface elevation on the mean water level to be zero:

$$
\int \eta \mathrm{d} x \mathrm{~d} y=0 \text { on MWL. }
$$

The boundary value problem (1) and (4)-(7) is solved analytically by separating the total velocity potential into three parts, namely, the rigid body motion, the liquid velocity induced by free surface elevation, and the StokesJoukowski potential. By integrating both sides of the equation and utilizing the orthogonality property, the 


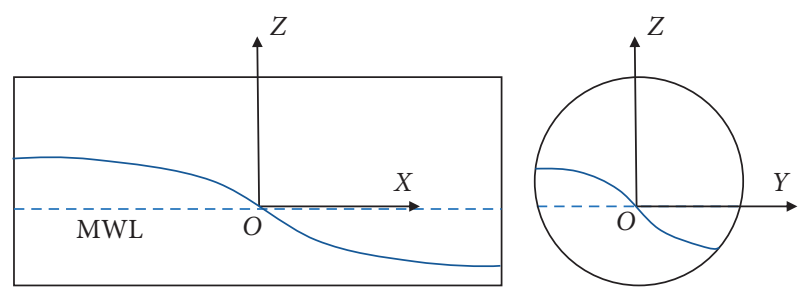

Figure 1: Coordinate system of linear sloshing problem.

sloshing in liquid tank can be expressed by a series of modal equations as [16]

$$
\begin{aligned}
\ddot{\beta}_{j}+\omega_{j}^{2} \beta_{j}= & K_{j}(t), \\
K_{j}(t)= & -\frac{\lambda_{1 j}}{\mu_{j}}\left(\ddot{\eta}_{1}(t)-g \eta_{5}(t)\right) \\
& -\frac{\lambda_{2 j}}{\mu_{j}}\left(\ddot{\eta}_{2}(t)+g \eta_{4}(t)\right)-\sum_{k=4}^{6} \frac{\ddot{\eta}_{k}(t) \lambda_{k j}}{\mu_{j}},
\end{aligned}
$$

where $\beta_{j}$ is a generalized coordinate, $\omega_{j}$ is the circular natural frequency of the $j$-th sloshing mode (note that $j$ can be an integer for $2 \mathrm{D}$ sloshing or an integer pair $j=(a, b)$ for $3 \mathrm{D}$ sloshing), and $\eta_{1}, \eta_{2}, \eta_{4}, \eta_{5}$, and $\eta_{6}$ denote the prescribed surge, sway, roll, pitch, and yaw motions of the sloshing tank, respectively. Equations (8) and (9) show that $\eta_{3}$, which denotes the heave motion of the tank, does not show up in $K_{j}(t)$, which means a pure heave motion will not induce liquid sloshing. However, in the coupled fluid-vehicle problems, if there is a coupling of heave motion with other DOFs in the vehicle motion equation, the heave motion still has to be considered and a 6-DOF equation of motion needs to be established. $\lambda_{i}$ and $\mu_{i}$ are the hydrodynamic coefficients corresponding to the $j$-th sloshing mode; $K_{j}(t)$ is jointly determined by external excitation and hydrodynamic properties of the fluid.

The hydrodynamic parameters corresponding to the $j$-th sloshing mode are given by [16]

$$
\begin{aligned}
& \mu_{j}=\frac{\rho g}{\omega_{j}^{2}} \int_{\sum_{0}} f_{j}^{2} \mathrm{~d} x \mathrm{~d} y, \\
& \lambda_{1 j}=\rho \int_{\sum_{0}} f_{j} x \mathrm{~d} x \mathrm{~d} y, \\
& \lambda_{2 j}=\rho \int_{\sum_{0}} f_{j} y \mathrm{~d} x \mathrm{~d} y, \\
& \lambda_{4 j}=\rho \int_{\sum_{0}} f_{j} \Omega_{4} \mathrm{~d} x \mathrm{~d} y, \\
& \lambda_{5 j}=\rho \int_{\sum_{0}} f_{j} \Omega_{5} \mathrm{~d} x \mathrm{~d} y .
\end{aligned}
$$

The hydrodynamic parameters are derived from the free surface shape function $f_{j}$ and the Stokes-Joukowski potential $\Omega_{i j}$, with the same scale of mass. The sloshing force and moment are calculated by solving the modal equation (equations (8) and (9)), given that all the prescribed motion and hydrodynamic parameters are known. Neglecting the sloshing moment induced purely by Stokes-Joukowski potential and considering only the effect of free surface vibration, the sloshing force and moment components can be written as [16]

$$
\begin{aligned}
& F_{1}(t)=-\sum_{j=1}^{\infty} \lambda_{1 j} \ddot{\beta}_{j}, \\
& F_{2}(t)=-\sum_{j=1}^{\infty} \lambda_{2 j} \ddot{\beta}_{j}, \\
& F_{3}(t)=0 \\
& M_{4}(t)=-\sum_{j=1}^{\infty}\left(g \lambda_{2 j} \beta_{j}+\lambda_{4 j} \ddot{\beta}_{j}\right), \\
& M_{5}(t)=-\sum_{j=1}^{\infty}\left(-g \lambda_{1 j} \beta_{j}+\lambda_{5 j} \ddot{\beta}_{j}\right), \\
& M_{6}(t)=-\sum_{j=1}^{\infty} \lambda_{6 j} \ddot{\beta}_{j} .
\end{aligned}
$$

Therefore, the fluid-induced force and moment can be decomposed into an inertial term that is equivalent to a solid mass, and a sloshing term induced by liquid vibration inside the tank.

\section{Frequency Domain Numerical Solution}

The velocity potential required for calculating the hydrodynamic coefficients in equations (10)-(14) can be solved numerically by frequency-domain boundary element method. A lot of highly efficient BEM programmes are available, most of which are designed to solve harmonic potential problems, apply nonhomogenous boundary conditions on the tank surface, and solve velocity potential induced by each degree of freedom of the rigid body motion [24]. In this way, the influence of fluid is simplified into added mass terms that can be incorporated into the rigid body motion to account for the sloshing effect, without introducing extra degrees of freedom. By taking advantage of Green's second identity and by using a fundamental solution that satisfies the Laplace equation (equation (1)), the velocity potential at any point within the fluid domain except for the source point is expressed as an integral on the wetted surface of the tank [25]:

$$
\Phi(P)=\int_{S}\left[\frac{\partial \Phi(Q)}{\partial n(Q)} G(P, Q)-\Phi(Q) \frac{\partial G(Q)}{\partial n(Q)}\right] \mathrm{d} S .
$$

The fundamental solution $G(P, Q)$ is the wave source function, determined by field point $P(x, y, z)$ and source point $Q\left(x_{0}, y_{0}, z_{0}\right)$, satisfying free surface boundary condition and may be written as $[26,27]$ 


$$
\begin{aligned}
(P, Q) & =\frac{1}{r}+\frac{1}{r^{\prime}}+\frac{2 K}{\pi} \int_{0}^{\infty} \mathrm{d} k \frac{(k+K)}{k \sinh k H-K \cosh k H} e^{-k H} J_{0}(k R) \\
r & =\sqrt{\left(x-x_{0}\right)^{2}+\left(y-y_{0}\right)^{2}+\left(z-z_{0}\right)^{2}} \\
r^{\prime} & =\sqrt{\left(x-x_{0}\right)^{2}+\left(y-y_{0}\right)^{2}+\left(z-z_{0}+2 H\right)^{2}}
\end{aligned}
$$

where $J_{0}$ is the Bessel function of zero-th order, $K$ is the infinite depth wave number, and $H$ is the water depth. Alternative forms of fundamental solution also exist, such as in $[28,29]$. The free-surface Green function for external domain as in equations (22)-(24) is presented here because the velocity potentials in the external and internal domain are solved together in the commercial program.

The velocity potential can be solved in both the time domain and frequency domain. For a time domain solution, a transient Green function has to be applied in order to take into account the memory effect of the fluid, which includes a convolution integral that has to be evaluated from the current time step back to a certain period of time [30]. A less time-consuming way is to solve the problem in frequency domain and convert the results to time domain by the inverse Fourier transform. Essentially, the approach regards the input excitation force and the resultant fluid response as the superposition of a series of harmonic components. In this way, the velocity potential at each calculated frequency will be considered as periodical:

$$
\Phi(x, y, z, t)=\operatorname{Re}\left(\Phi(x, y, z) e^{-i \omega t}\right) .
$$

If the 6-DOF external excitation $\eta_{\mathrm{i}}$ is periodical, i.e.,

$$
\eta_{i}=\operatorname{Re}\left(A_{i} e^{-i \omega t}\right)
$$

where $\eta_{i}=\left(\eta_{1}, \eta_{2}, \eta_{4}, \eta_{5}, \eta_{6}\right)^{\mathrm{T}}$ is the 5 components of the prescribed tank motion. Then, the modal response $\beta_{m}$ can be written as [16]

$$
\begin{aligned}
\ddot{\beta}_{m}= & P_{m} \ddot{\eta}_{i}, \\
P_{m}= & \frac{\omega^{2}}{\omega_{m}^{2}-\omega^{2}}\left(-\frac{\lambda_{1 m}}{\mu_{m}},-\frac{\lambda_{2 m}}{\mu_{m}}, \frac{1}{\omega^{2}} \frac{g \lambda_{2 m}}{\mu_{m}}\right. \\
& \left.-\frac{\lambda_{4 m}}{\mu_{m}},-\frac{1}{\omega^{2}} \frac{g \lambda_{1 m}}{\mu_{m}}-\frac{\lambda_{5 m}}{\mu_{m}},-\frac{\lambda_{6 m}}{\mu_{m}}\right),
\end{aligned}
$$

where $\lambda_{1 m}$ is the hydrodynamic coefficient. The resultant sloshing force and moment induced by the $m$-th mode may be written as

$$
F_{m}=-\left(M \ddot{\eta}_{i}+Q_{m} \ddot{\beta}_{m}\right)=-(M+A) \ddot{\eta}_{i}
$$

where $\beta_{m}$ is the modal displacement, sloshing force $F_{m}$ is written in $5 \times 5$ matrix form, and $M$ is the liquid mass matrix considered as solid. $Q_{m}$ is the transfer function between modal response $\beta$ and the sloshing forces in $5 \times 1$ matrix form, which can be summarized from equations (15)-(20):

$$
Q_{m}=\left(-\lambda_{1 m},-\lambda_{2 m}, \frac{g \lambda_{2 m}}{\omega^{2}}-\lambda_{4 m},-\frac{g \lambda_{1 m}}{\omega^{2}}-\lambda_{5 m},-\lambda_{6 m}\right)^{\mathrm{T}} .
$$

Equation (25) shows that if the excitation is periodical, the effect of sloshing is like changing the mass of the body by an amount of $A$. Thus the matrix $A$ is called added mass matrix. The added mass can be either positive or negative. Note that when the added mass is negative, the "resultant" mass of the tank becomes lighter which makes the tank more likely to capsize. If more than one sloshing modes are considered, the total added mass should be the sum of the modal component corresponding to every sloshing mode. The added mass can be calculated by the integration of total radiation potential at the tank wetted surface [25]:

$$
A_{i j}=\rho \iint_{S} \varphi_{j} n_{i} \mathrm{~d} S
$$

Note that the off-diagonal terms $A_{i j}$ means the effect of $i$ th DOF on $j$-th DOF, which is the coupling effect between different DOFs. $\varphi_{j}$ is the radiation potential component of the $j$-th mode, while $n_{i}$ is the normal vector of the $i$-th mode. In marine applications, the added mass terms is incorporated into the total mass of the marine structure, so that the water effect is fully described by added mass alone, without additional DOFs to the motion equation. The wave load has a spectral nature and as a result a steady-state solution can be achieved. However, the excitation due to vehicle operation such as sudden braking is highly transient. The duration of the acceleration can be several seconds only. Since the discontinuous and transient excitation make it difficult to apply the spectral method and Fourier transform, the added mass calculated for steady-state sloshing cannot be directly used in transient sloshing analysis. In view of this, we explore the possibility of utilizing existing hydrodynamics software for use in marine engineering, with some modification and postprocessing, to solve the transient sloshing problem under vehicle operation. Alternatively, one may also compute the hydrodynamic coefficients by solving the eigenvalues and eigenfunctions of the homogenous boundary value problem by changing equation (6) into a homogenous boundary condition.

From equations (25)-(30), it can be found that the added mass terms are functions of the hydrodynamic parameters $\lambda_{1 m}, \lambda_{2 m}$, and $\mu_{m}$ and natural frequency $\omega_{m}$, in the following form [16]:

$$
\begin{aligned}
& A_{11}=\sum_{m=(1,0)}^{\infty} \frac{\lambda_{1 m}^{2}}{\mu_{m}} \frac{\omega^{2}}{\omega_{m}^{2}-\omega^{2}}, \\
& A_{22}=\sum_{m=(0,1)}^{\infty} \frac{\lambda_{2 m}^{2}}{\mu_{m}} \frac{\omega^{2}}{\omega_{m}^{2}-\omega^{2}}, \\
& A_{15}=\sum_{m=(1,0)}^{\infty}\left(\frac{g \lambda_{1 m}}{\omega^{2}}+\lambda_{5 m}\right) \frac{\lambda_{1 m}}{\mu_{m}} \frac{\omega^{2}}{\omega_{m}^{2}-\omega^{2}}
\end{aligned}
$$




$$
A_{24}=\sum_{m=(0,1)}^{\infty}\left(-\frac{g \lambda_{2 m}}{\omega^{2}}+\lambda_{4 m}\right) \frac{\lambda_{2 m}}{\mu_{m}} \frac{\omega^{2}}{\omega_{m}^{2}-\omega^{2}}
$$

Note that the above added masses are induced by the liquid sloshing mode only.

\section{Sloshing in Cylindrical Tank during Turning or Braking}

4.1. Derivation of Hydrodynamic Coefficients from Added Mass. From equations (32)-(35), it can be seen that five hydrodynamic coefficients $\lambda_{1 m}, \lambda_{2 m}, \lambda_{4 m}, \lambda_{5 m}$, and $\mu_{m}$ are related to four added mass terms $A_{11}, A_{22}, A_{15}$, and $A_{24}$. In order to calculate these hydrodynamic coefficients using the added mass results, the free surface shape of each vibration mode has to be assumed as an extra equation, or there will be one more unknown equation than established equations. For longitudinal sloshing, the free surface shape is in sinusoidal form. But for lateral sloshing in cylindrical tanks, the free surface shape does not have a simple analytical form. In the following analysis, we assume that the free surface of lateral sloshing is still a trigonometric function, which is proved to be accurate enough, though it does not strictly satisfy boundary conditions in equations (4)-(6).

If the lowest mode only is considered while all other higher modes are neglected, the hydrodynamic coefficients are calculated using added mass at infinite frequency. With the free surface shape known, the required hydrodynamic coefficients can be solved using equations (10)-(14) and (32)-(35). If higher order modes are considered as well, the added mass contributed by each sloshing mode needs to be calculated by fitting the numerical results to the analytical function (equations (32)-(35)). The commercial BEM program HydroSTAR will be used in the following studies for calculating added mass terms. It is expected that higher order modes will have lesser influence on the values of the added mass. Only the first three sloshing modes are employed for calculation of hydrodynamic coefficients $(\lambda$ and $\mu$ ), sloshing forces, and moment.

\subsection{Comparison between Analytical and Numerical Results.} Consider the case of a partially filled rectangular tank where analytical solution is available in [16]. The tank has a length $L=8.0 \mathrm{~m}$, width $B=2.0 \mathrm{~m}$, and a liquid filling depth $d=0.8 \mathrm{~m}$. The numerical model of the rectangular tank is shown in Figure 2. The origin of the coordinate system is at the centre of the rectangle on the mean water level, and the $x$ direction is along the length of the rectangle, as can be seen in Figure 2. The numerical model consists of 470 panel elements. It can be seen from the analytical solution to the added mass as expressed in equations (32)-(35) that added mass becomes infinite at sloshing natural frequencies. To avoid the singularity problem, an artificial damping term is added to the velocity potential by adding an imaginary term to the boundary condition on the tank wall according to [25] in the following form:

$$
\frac{\partial \phi}{\partial n}=v_{n}+i \varepsilon k_{0} \phi
$$

where $\varepsilon$ is an arbitrary damping coefficient. The simulation is performed within a frequency range from $0.1 \mathrm{rad} / \mathrm{s}$ to $5.0 \mathrm{rad} / \mathrm{s}$, which is deemed large enough to capture the fundamental sloshing frequencies of concern. Added mass properties are determined at a frequency of $50.0 \mathrm{rad} / \mathrm{s}$ which is deemed far away from the sloshing periods so that the effect of the damping is negligible. Figure 3 shows the added mass $A_{11}$ and $A_{22}$ with respect to the excitation frequency with the inclusion of viscous damping. The results show that in the calculated frequency range, three longitudinal natural sloshing frequencies are captured at $\omega=1.05 \mathrm{rad} / \mathrm{s}, 2.95 \mathrm{rad} /$ $\mathrm{s}$, and $4.25 \mathrm{rad} / \mathrm{s}$, while only one lateral natural frequency is found to be prominent at $3.65 \mathrm{rad} / \mathrm{s}$. It should be noted that for longitudinal sloshing, higher order sloshing modes also have contribution to the added mass while lateral sloshing is predominated by the fundamental sloshing mode.

The hydrodynamic coefficients required for calculating transient sloshing are then evaluated based on the added mass results and compared with available analytical solution reported in [16]. Table 1 shows the hydrodynamic coefficients when only the fundamental sloshing mode is considered. It can be seen from Table 1, the results obtained numerically match well with those by analytical solution. Some minor differences exist which may be explained as due to the neglect of the 3D effect in the numerical model. Due to the effect of higher order modes, the parameters of the longitudinal sloshing have more difference than those of the lateral sloshing when compared to analytical solution.

When considering the two higher order modes $m=(1,0)$ and $m=(3,0)$ for longitudinal sloshing and fitting the added mass data with the added mass expression with three components, the hydrodynamic parameter results are closer to the analytical results with $\lambda_{1(1,0)}=-23505 \mathrm{~kg}$ and $\lambda_{5(1,0)}=13907 \mathrm{kgm}$. The magnitude of $\lambda_{1(1,0)}$ and $\mu_{(1,0)}$ is reduced by ruling out the contribution of higher order sloshing modes.

4.3. Longitudinal Sloshing Induced by Braking. In this section, the liquid sloshing in horizontal cylindrical tank modelled as shown in Figure 4 is studied. Numerical method has to be applied in order to calculate the natural frequency and hydrodynamic coefficients of this tank geometry. As stated in Section 4.1, the hydrodynamic coefficients are calculated by fitting equations (32)-(35) into the added mass output, with a prescribed free surface shape function. Cosine function is chosen as a free surface shape function in this case because for longitudinal sloshing it satisfies the boundary condition in equation (7). The validation case of sloshing during braking refers to [22], in which the tank is subject to a $0.1 \mathrm{~g}$ deceleration with a linear ramp function from $t=0.0 \mathrm{~s}$ to $t=2.0 \mathrm{~s}$. The sloshing force and moment are then calculated using a direct BEM method and CFD simulation. The tank has a length $L=5.75 \mathrm{~m}$ and radius $R=1.2 \mathrm{~m}$. The tank is filled with water at two filling levels: $h_{1}=1.0 R$ and $1.5 R$. The water is assumed to have a density of $1000 \mathrm{~kg} / \mathrm{m}^{3}$ and low viscosity. The sloshing force and moment in three and half cycles of the natural period are calculated. 


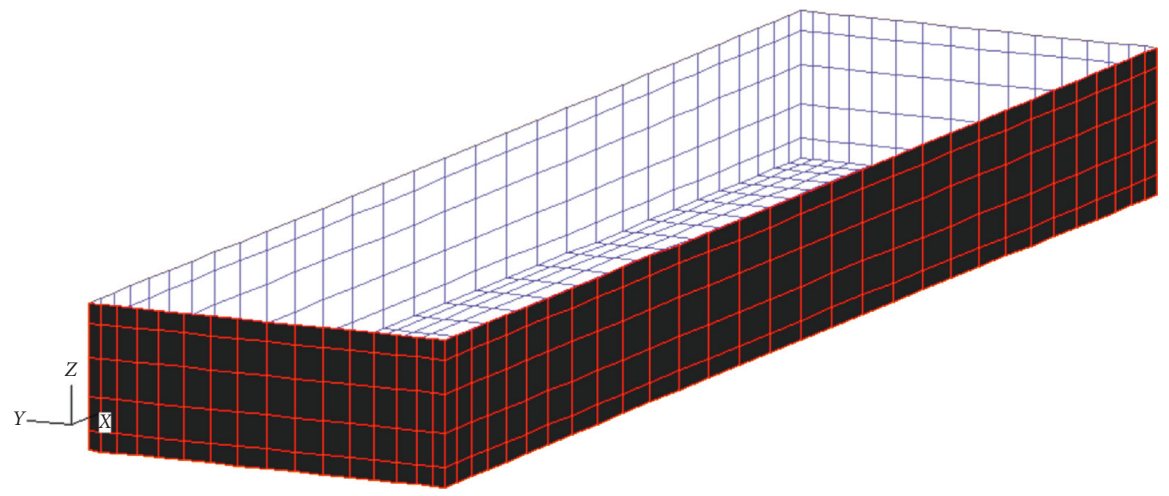

Figure 2: Numerical model of rectangular tank.

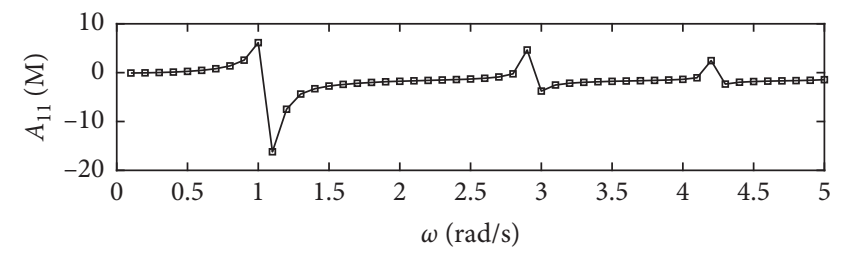

(a)

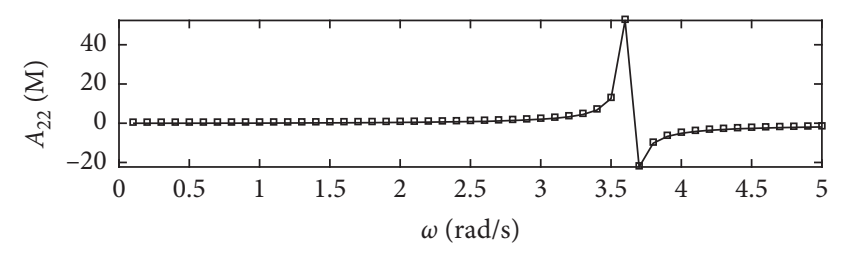

(b)

Figure 3: Added mass calculated by HydroSTAR: (a) $A_{11}$; (b) $A_{22}$.

TABLE 1: Hydrodynamic coefficients calculated by analytical and numerical methods.

\begin{tabular}{lccccc}
\hline Parameter & Lateral & & Parameter & \multicolumn{2}{c}{ Longitudinal } \\
& Analytical & Numerical & 3.65 & $\omega_{(1,0)}$ & 1.08 \\
Numerical \\
$\omega_{(0,1)}$ & 3.62 & -5764 & $\lambda_{1(1,0)}$ & -22047 & -265 \\
$\lambda_{2(0,1)}$ & -5512 & 5231 & $\mu_{(1,0)}$ & 56920 & 72982 \\
$\mu_{(0,1)}$ & 5092 & -1.102 & $K_{t(1,0)}$ & -0.387 & -0.365 \\
$K_{t(0,1)}$ & -1.082 & -3848 & $\lambda_{5(1,0)}$ & 17494 & 20052 \\
$\lambda_{4(0,1)}$ & -3908 & & &
\end{tabular}

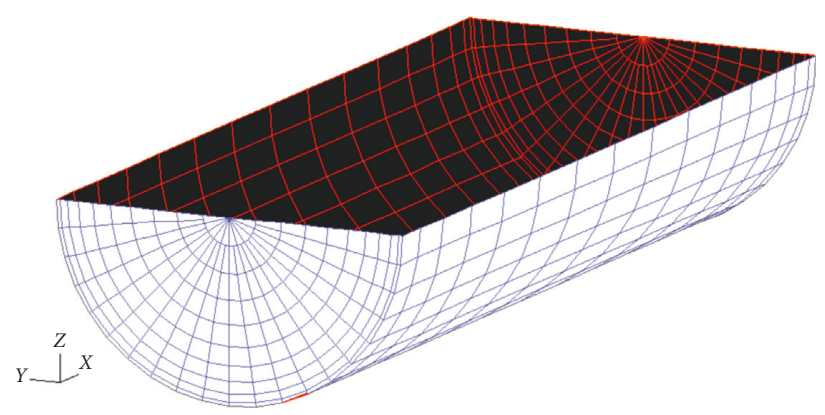

(a)

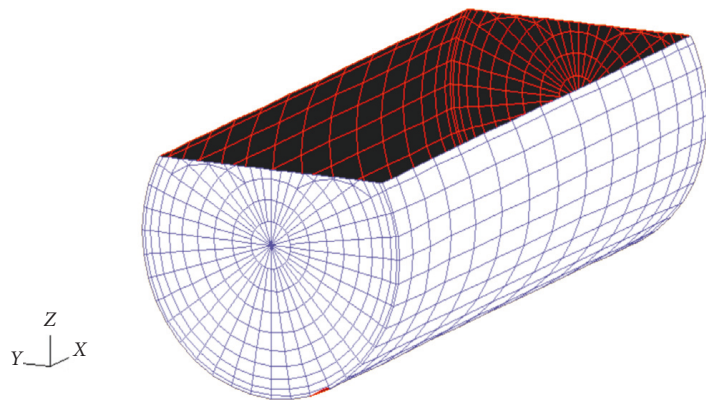

(b)

FIgURE 4: Numerical model of horizontal circular tank: (a) $h_{l}=1.0 R$; (b) $h_{1}=1.5 R$.

The numerical model is created using the same program in a similar manner. 680 panels are used for the tank with $h_{1}=0.5 R$ while 1076 panels are used for the case with $h_{1}=1.5 R$. The results of added mass are shown in Figure 5. For both filling levels, the second sloshing mode has a prominent effect on the distribution of added mass. For $50 \%$ filling level, the first two sloshing frequencies are $1.65 \mathrm{rad} / \mathrm{s}$ and $3.75 \mathrm{rad} / \mathrm{s}$. They become $2.05 \mathrm{rad} / \mathrm{s}$ and $4.15 \mathrm{rad} / \mathrm{s}$ for $h_{1}=1.5 R$.
Table 2 shows the hydrodynamic coefficients deducted from added mass results. Compared with a half-filled tank, the tank with a filling level $h_{1}=1.5 R$ has a significantly higher mass, while the virtual mass is not increased proportionally. As a result, the hydrodynamic parameters are only slightly increased as well. Consequently, it is expected that the sloshing with $h_{1}=1.5 R$ should have a smaller dynamic amplification factor. Figure 6 shows the time history results of the liquid force. Also plotted in this figure are the results 


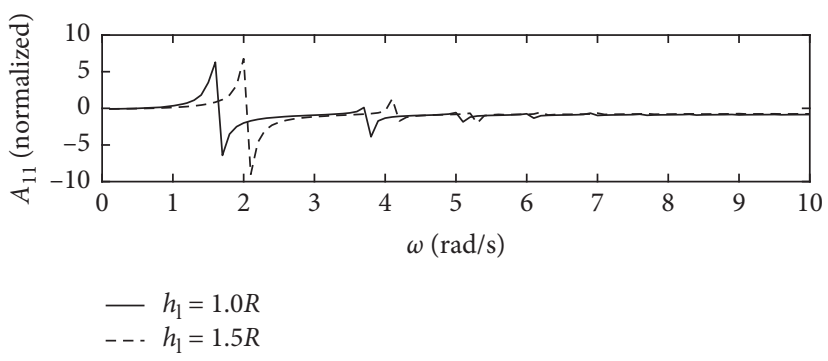

(a)

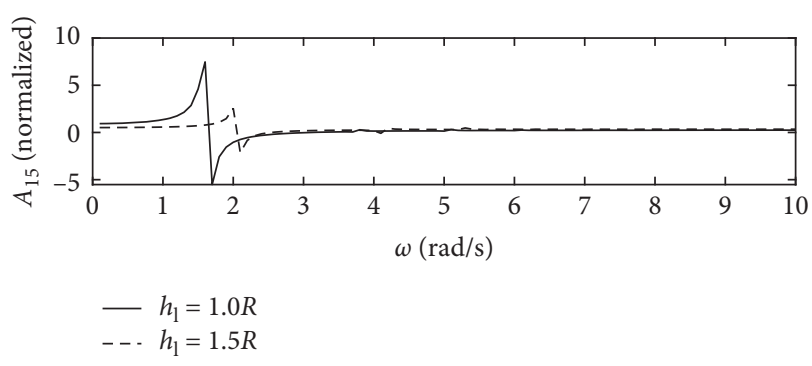

(b)

Figure 5: Added mass calculated by HydroSTAR: (a) $A_{11}$; (b) $A_{15}$.

TABLE 2: Hydrodynamic coefficients calculated based on added mass.

\begin{tabular}{lccc}
\hline Parameter & Unit & $h_{1}=1.0 R$ & $h_{1}=1.5 R$ \\
\hline$M$ & $\mathrm{~kg}$ & 13000 & 20860 \\
$\omega_{(1,0)}$ & $\mathrm{rad} / \mathrm{s}$ & 1.65 & 2.10 \\
$A_{11}$ & $\mathrm{~kg}$ & -10660 & -14924 \\
$A_{15}$ & $\mathrm{kgm}$ & 9806 & 21022 \\
$\mu_{(1,0)}$ & $\mathrm{kg}$ & 25434 & 13571 \\
$\lambda_{1(1,0)}$ & $\mathrm{kgm}$ & -16465 & -14231 \\
\hline
\end{tabular}

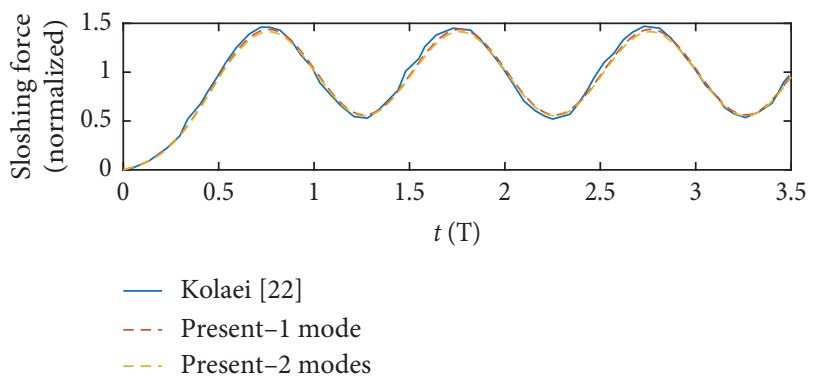

(a)

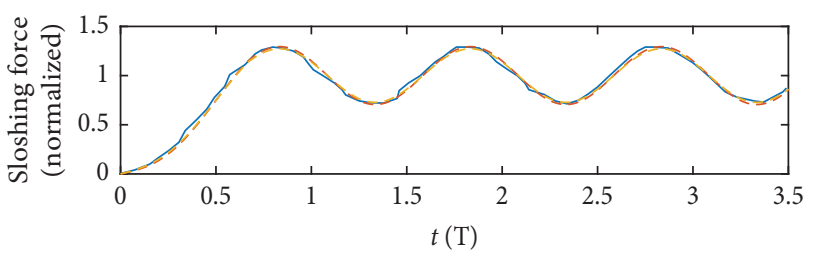

$\begin{array}{ll}\text { _ } & \text { Kolaei [22] } \\ \text { _- } & \text { Present-1 mode } \\ \text { _. } & \text { Present-2 modes }\end{array}$

(b)

FIGURE 6: Comparison of sloshing forces: (a) $h_{1}=1.0 R$; (b) $h_{1}=1.5 R$.

reported in [22]. Note that in Figure 6 the time and force are normalized with respect to fundamental sloshing period and the liquid force corresponding to the rigid body motion, respectively. It can be seen from Figure 6 that the sloshing forces predicted by the proposed method with only the first order sloshing mode agree well with those in [22]. The inclusion of the second order sloshing mode is found to contribute only $1.7 \%$ to the total liquid force for both $h_{1}=1.0 R$ and $h_{1}=1.5 R$. It is also found that a higher filling level leads to a larger increase in the inertia effect than the free surface sloshing effect. The moment dynamic amplification factor (DAF) is found to be 1.44 for $h_{1}=1.5 R$ and 1.64 for $h_{1}=1.0 R$. Note that DAF is defined as a ratio of the maximum response to the mean response.

4.4. Lateral Sloshing Induced by Vehicle Turning. The above case shows that the adoption of the free surface shape corresponding to a rectangular tank for a horizontally placed cylindrical tank is able to yield satisfactory results. The case of a vehicle carrying a half-filled cylindrical tank subjected to the operation of switching rail as reported in [17], is next considered. Unlike the above cases, the cosine function no longer satisfies the boundary condition at body surface, but as an odd function, it still fulfils the requirement of mass conservation. A free surface shape has to be assumed in order to calculate the hydrodynamic coefficients. In the following case, two free surface shapes represented with: (1) a cosine function and (2) a linear function $f_{(0,1)}=-x / R$ are applied in order to investigate the influence of the shape function on the sloshing force results. Figure 7 shows the centrifugal acceleration of the vehicle during the turning. The tank has a length $L=15.5 \mathrm{~m}$ and a radius $R=1.5 \mathrm{~m}$. The liquid inside the tank is gasoline with density of $820 \mathrm{~kg} / \mathrm{m}^{3}$ Note that all the parameters listed above are the same as those reported in [17].

The modal displacement, acceleration, and liquid force and moment are shown in Figure 8. Compared with the results from cosine free surface shape, the sloshing force and moment calculated based on the linear shape function remain identical, and these two trial functions result in a similar accuracy. As can be seen in Figure 8, the results generated using the proposed model agree reasonably well 


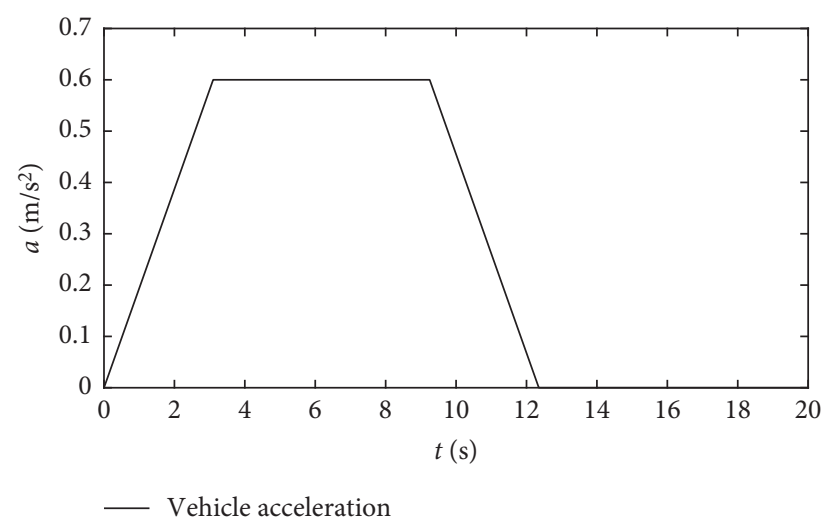

Figure 7: Acceleration curve during vehicle turning.

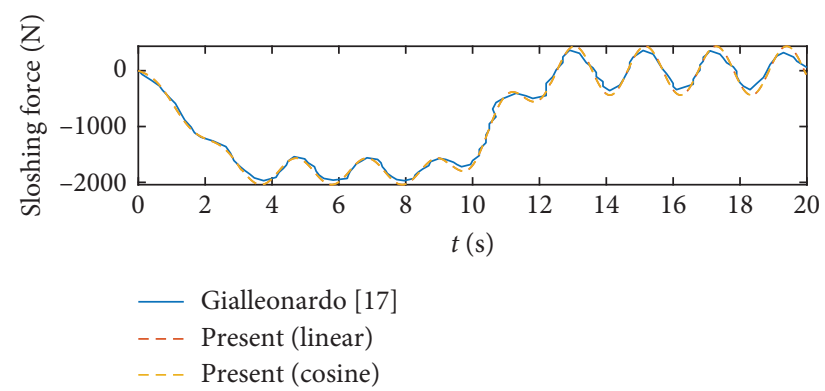

(a)

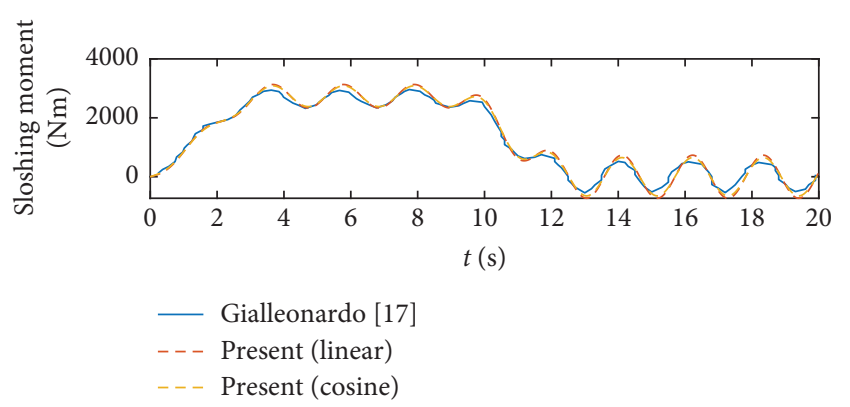

(b)

FIgURe 8: Comparison of sloshing force and moment: (a) sloshing; (b) sloshing moment.

with available CFD results reported in [17]. Although there are some minor discrepancies between the current results and CFD simulations, the difference is well within the acceptable engineering tolerance. Moreover, the current results are on the conservative side.

4.5. Sloshing Induced by Vehicle Pitching Motion. The aforementioned investigations examined the accuracy of the proposed liquid sloshing model for a partially filled tank subject to longitudinal or centrifugal accelerations. In reality, road tankers also experience pitching motions due to the existence of road bumps and/or road roughness. The pitching motion of a vehicle can also induce the liquid sloshing (see equation (9)), and its effect on the liquid motion is worth investigation. In this section, a rectangular tank with length $L=0.92 \mathrm{~m}$, width $W=0.46 \mathrm{~m}$, and height $H=0.62 \mathrm{~m}$ subjected to a periodical pitching excitation with two amplitudes, namely, 4 degrees and 8 degrees, are considered. Note that this represents the case of a sloshing tank undergoing a shaking table test as reported in [31]. The output of the model test is the fluctuating pressure on the vertical walls, measured by pressure sensors. The dimension of the rectangular tank is $0.92 \mathrm{~m}$ by $0.46 \mathrm{~m}$ by $0.62 \mathrm{~m}$. The pressure sensor is located at the side wall $6 \mathrm{~cm}$ above the tank bottom. Different filling levels are tested provided a prescribed tank pitch amplitude of 4 degrees or 8 degrees. During the liquid sloshing motion, the hydrodynamic pressure at any point in the fluid field under pure pitch excitation is given by [16]

$$
p=p_{0}-\rho g z+\rho g x \eta_{5}-\rho \Omega_{5}(x, y, z) \ddot{\eta}_{5}-\rho \sum_{i=1}^{\infty} \ddot{\beta}_{i} \kappa_{i}^{-1} \varphi_{i}(x, y, z) \text {, }
$$

where $\Omega_{5}$ is the lateral component of the StokesJoukowski potential and $\varphi_{(x, y, z)}$ is the velocity potential induced by free surface vibration. It is easy to observe that the total pressure comprises a hydrostatic component $\left(p_{0^{-}}\right.$ $\rho g z$ ) and a hydrodynamic component induced by sloshing. Since the tank is rectangular, the analytical solution is directly adopted here to validate the output of linear modal equation. Figure 9(a) shows the pressure measurements inside a tank with $25 \%$ filling level subject to a harmonic excitation with a circular frequency of $2.0 \mathrm{rad} / \mathrm{s}$. It can be seen that the numerical results match well with the experimental test results, thereby validating the current model accounting for the liquid sloshing induced by the pitch motion of the tank.

The case of a half-filled tank subject to harmonic pitching excitation is next examined, as shown in Figure 9(b). It can be seen that when the amplitude of the excitation is as small as 4 degrees, the linear numerical results agree very well with the model test results. When the amplitude of the pitch is increased to 8 degrees, the results are found to be overestimated by the numerical 


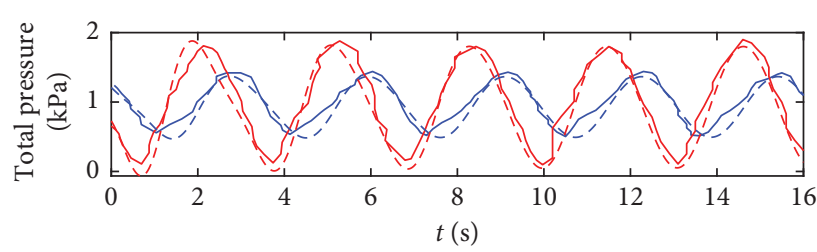

Test (2005)-4 deg
Test (2005)-8 deg

- - Present-8 deg

(a)

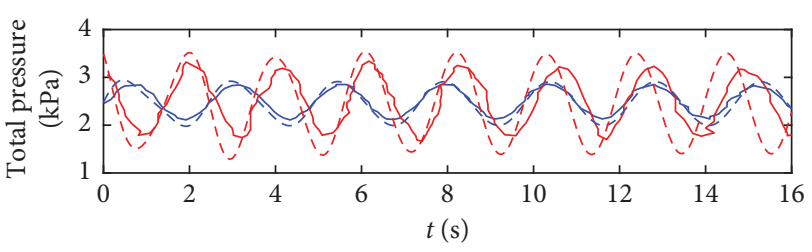

- Test (2005)-4 deg _- - Present-4 deg

- Test (2005)-8 deg

(b)

FiguRE 9: Comparison of pressure on tank wall: (a) $h_{1}=0.5 R$; (b) $h_{1}=1.0 R$.

model. This may be explained as due to the nonlinearity of the sloshing inside the tank when the excitation is considered large enough, since with 8 degree pitching, irregularities show up in the period and amplitude of the measured total pressure, which implies nonlinearity.

\section{Liquid Sloshing under Combined Translational and Rotational Excitations}

In order to investigate the sloshing behaviour inside a tank under combined translational and rotational excitations, a vehicle carrying a partially filled horizontal cylindrical tank passing a road bump or a road pit when subjecting to a braking is next investigated. In this case, an articulated tractor-semitrailer representing a road tanker carrying liquid cargo is considered as shown in Figure 10. The components of the vehicle are modelled as rigid bodies and the suspension systems are idealized using spring-dashpot units. By neglecting the interaction between vehicle components in the longitudinal direction and assuming that the vertical displacement of tyres is following the road surface profile [32], the equations of motion governing the vertical and pitch DOFs of the tractor and semitrailer as well as the liquid sloshing model can be written as

$$
\begin{aligned}
& M_{\mathrm{t}} \ddot{z}_{\mathrm{t}}+\left(2 c_{\mathrm{w}}+c_{\mathrm{bt}}\right) \dot{z}_{t}+\left(-c_{\mathrm{w}} l_{\mathrm{w} 1}+c_{w} l_{\mathrm{w} 2}+c_{\mathrm{bt}} l_{\mathrm{bt}}\right) \dot{\theta}_{t}-c_{\mathrm{bt}} \dot{z}_{\mathrm{s}}+c_{\mathrm{bt}} l_{\mathrm{bs}} \dot{\theta}_{\mathrm{s}}-c_{\mathrm{w}} \dot{z}_{\mathrm{w} 1}-c_{\mathrm{w}} \dot{z}_{\mathrm{w} 2}+\left(2 k_{\mathrm{w}}+k_{\mathrm{bt}}\right) z_{\mathrm{t}} \\
& +\left(-k_{\mathrm{w}} l_{\mathrm{w} 1}+k_{\mathrm{w}} l_{\mathrm{w} 2}+k_{\mathrm{bt}} l_{\mathrm{bt}}\right) \theta_{\mathrm{t}}-k_{\mathrm{bt}} z_{\mathrm{s}}+k_{\mathrm{bt}} l_{\mathrm{bs}} \theta_{\mathrm{s}}-k_{\mathrm{w}} z_{\mathrm{w} 1}-k_{\mathrm{w}} z_{\mathrm{w} 2}=-M_{\mathrm{t}} g \\
& I_{\mathrm{t}} \ddot{\theta}_{\mathrm{t}}+\left(-c_{\mathrm{w}} l_{\mathrm{w} 1}+c_{\mathrm{w}} l_{\mathrm{w} 2}+c_{\mathrm{bt}} l_{\mathrm{bt}}\right) \dot{z}_{\mathrm{t}}+\left(-c_{\mathrm{w}} l_{\mathrm{w} 1}^{2}+c_{\mathrm{w}} l_{\mathrm{w} 2}^{2}+c_{\mathrm{bt}} l_{\mathrm{bt}}^{2}\right) \dot{\theta}_{\mathrm{t}}-c_{\mathrm{bt}} l_{\mathrm{bt}} \dot{z}_{\mathrm{s}}+c_{\mathrm{bt}} l_{\mathrm{bs}} l_{\mathrm{bt}} \dot{\theta}_{\mathrm{s}}+c_{\mathrm{w}} l_{\mathrm{w} 1} \dot{z}_{\mathrm{w} 1}-c_{\mathrm{w}} l_{\mathrm{w} 2} \dot{z}_{\mathrm{w} 2} \\
& +\left(-k_{\mathrm{w}} l_{\mathrm{w} 1}+k_{\mathrm{w}} l_{\mathrm{w} 2}+k_{\mathrm{bt}} l_{\mathrm{bt}}\right) z_{\mathrm{t}}+\left(-k_{\mathrm{w}} l_{\mathrm{w} 1}^{2}+k_{\mathrm{w}} l_{\mathrm{w} 2}^{2}+k_{\mathrm{bt}} l_{\mathrm{bt}}^{2}\right) \theta_{\mathrm{t}}-k_{\mathrm{bt}} l_{\mathrm{bt}} z_{\mathrm{s}}+k_{\mathrm{bt}} l_{\mathrm{bs}} l_{\mathrm{bt}} \theta_{\mathrm{s}}+k_{\mathrm{w}} l_{\mathrm{w} 1} z_{\mathrm{w} 1}-k_{\mathrm{w}} l_{\mathrm{w} 2} z_{\mathrm{w} 2}=0 \\
& \left(M_{\mathrm{s}}+M_{\mathrm{l}}\right) \ddot{z}_{\mathrm{s}}-c_{\mathrm{bt}} \dot{z}_{\mathrm{t}}-c_{\mathrm{bt}} l_{\mathrm{bt}} \dot{\theta}_{\mathrm{t}}+\left(2 c_{\mathrm{w}}+c_{\mathrm{bt}}\right) \dot{z}_{\mathrm{s}}+\left(c_{\mathrm{w}} l_{\mathrm{w} 3}+c_{\mathrm{w}} l_{\mathrm{w} 4}-c_{\mathrm{bt}} l_{\mathrm{bs}}\right) \dot{\theta}_{\mathrm{s}}-c_{\mathrm{w}} \dot{z}_{\mathrm{w} 3}-c_{\mathrm{w}} \dot{z}_{\mathrm{w} 4}-k_{\mathrm{bt}} z_{\mathrm{t}}-k_{\mathrm{bt}} l_{\mathrm{bt}} \theta_{\mathrm{t}} \\
& +\left(2 k_{\mathrm{w}}+k_{\mathrm{bt}}\right) z_{\mathrm{s}}+\left(k_{\mathrm{w}} l_{\mathrm{w} 3}+k_{\mathrm{w}} l_{\mathrm{w} 4}-k_{\mathrm{bt}} l_{\mathrm{bs}}\right) \theta_{\mathrm{s}}-k_{\mathrm{w}} z_{\mathrm{w} 3}-k_{\mathrm{w}} z_{\mathrm{w} 4}=-\left(M_{\mathrm{s}}+M_{\mathrm{l}}\right) g \\
& \left(I_{\mathrm{s}}+I_{1}\right) \ddot{\theta}_{\mathrm{s}}+\lambda_{5(1,0)} \ddot{\beta}_{(1,0)}+c_{\mathrm{bt}} l_{\mathrm{bs}} \dot{z}_{\mathrm{t}}+c_{\mathrm{bt}} l_{\mathrm{bs}} l_{\mathrm{bt}} \dot{\theta}_{\mathrm{t}}+\left(-c_{\mathrm{bt}} l_{\mathrm{bs}}+c_{\mathrm{w}} l_{\mathrm{w} 3}+c_{\mathrm{w}} l_{\mathrm{w} 4}\right) \dot{z}_{\mathrm{s}} \\
& +\left(c_{\mathrm{bt}} l_{\mathrm{bs}}^{2}+c_{\mathrm{w}} l_{\mathrm{w} 3}^{2}+c_{\mathrm{w}} l_{\mathrm{w} 4}^{2}\right) \dot{\theta}_{\mathrm{s}}-c_{\mathrm{w}} l_{\mathrm{w} 3} \dot{z}_{\mathrm{w} 3}-c_{\mathrm{w}} l_{\mathrm{w} 4} \dot{z}_{\mathrm{w} 4}+k_{\mathrm{bt}} l_{\mathrm{bs}} z_{\mathrm{t}} \\
& +k_{\mathrm{bt}} l_{\mathrm{bs}} l_{\mathrm{bt}} \theta_{\mathrm{t}}+\left(-k_{\mathrm{bt}} l_{\mathrm{bs}}+k_{\mathrm{w}} l_{\mathrm{w} 3}+k_{\mathrm{w}} l_{\mathrm{w} 4}\right) z_{\mathrm{s}}+\left(k_{\mathrm{bt}} l_{\mathrm{bs}}^{2}+k_{\mathrm{w}} l_{\mathrm{w} 3}^{2}+k_{\mathrm{w}} l_{\mathrm{w} 4}^{2}\right) \theta_{\mathrm{s}}-k_{\mathrm{w}} l_{\mathrm{w} 3} z_{\mathrm{w} 3}-k_{\mathrm{w}} l_{\mathrm{w} 4} z_{\mathrm{w} 4} \\
& -g \lambda_{1(1,0)} \beta_{(1,0)}=-M_{1} d_{1} a+M_{1} g l_{1}
\end{aligned}
$$

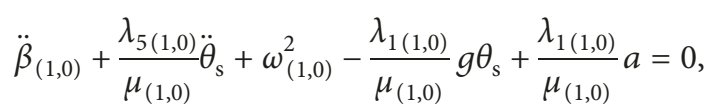

where $M_{\mathrm{t}}, M_{\mathrm{s}}$, and $M_{\mathrm{l}}$ refer to the mass of the tractor, unladen semitrailer, and liquid cargo, respectively; $I_{\mathrm{t}}, I_{\mathrm{s}}$, and $I_{1}$ denote the mass moment of inertia of the tractor, semitrailer, and liquid cargo, respectively; $c_{\mathrm{w}}, c_{\mathrm{bt}}$ and $k_{\mathrm{w}}, k_{\mathrm{bt}}$ are the damping and stiffness coefficients of the suspension systems connecting the wheels and the vehicle bodies as well as the connection between the tractor and the semitrailer, respectively; $l_{\mathrm{w} 1}$ and $l_{\mathrm{w} 2}$ are the horizontal distances from the 


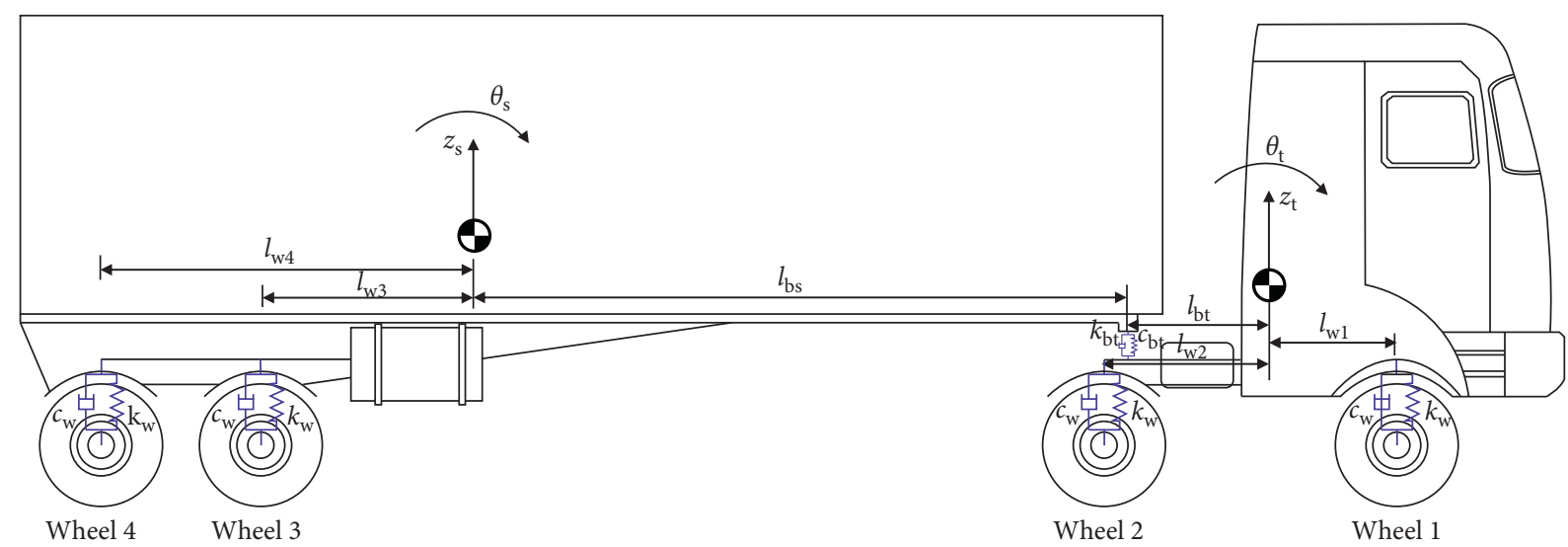

Figure 10: Schematic drawing of vehicle model.

mass centre of the tractor to wheel 1 and wheel 2, respectively; likewise, $l_{\mathrm{w} 3}$ and $l_{\mathrm{w} 4}$ are the horizontal distances from the mass centre of the semitrailer to wheel 3 and wheel 4 , respectively; $l_{\mathrm{bt}}$ and $l_{\mathrm{bs}}$ are the horizontal distances from the tractor-semitrailer connection point to the mass centres of the tractor and semitrailer, respectively; $l_{1}$ and $d_{1}$ are the horizontal and vertical distances between the mass centres of the liquid cargo and semitrailer, respectively; $z_{\mathrm{t}}, z_{\mathrm{s}}$ and $\theta_{\mathrm{t}}$ and $\theta_{\mathrm{s}}$ are the vertical and pitch degrees of freedom of the tractor and semitrailer, respectively; and $a$ is the longitudinal acceleration of the vehicle.

The vehicle with an initial velocity $V_{0}$ begins to decelerate at a distance $x_{0}$ from the bump at until the vehicle velocity becomes zero. The length of the cylindrical tank is $L$, and the road bump or road pit is represented by a parabolic arch with a span of $s$ and a height $h$. The geometry suggests that the vertical displacement of four wheels when travelling on the road bump can be written as (for $s=3.7 \mathrm{~m}$ )

$$
\begin{aligned}
z_{\mathrm{w} 1}= & -\frac{400}{1369} h\left(V_{0} t+\frac{1}{2} a t^{2}-x_{0}\right)^{2}+\frac{40}{37} h\left(V_{0} t+\frac{1}{2} a t^{2}-x_{0}\right), \\
z_{\mathrm{w} 2}= & -\frac{400}{1369} h\left(V_{0} t+\frac{1}{2} a t^{2}-L_{\mathrm{w} 12}-x_{0}\right)^{2} \\
& +\frac{40}{37} h\left(V_{0} t+\frac{1}{2} a t^{2}-L_{\mathrm{w} 12}-x_{0}\right), \\
z_{\mathrm{w} 3}= & -\frac{400}{1369} h\left(V_{0} t+\frac{1}{2} a t^{2}-L_{\mathrm{w} 13}-x_{0}\right)^{2} \\
& +\frac{40}{37} h\left(V_{0} t+\frac{1}{2} a t^{2}-L_{\mathrm{w} 13}-x_{0}\right), \\
z_{\mathrm{w} 4}= & -\frac{400}{1369} h\left(V_{0} t+\frac{1}{2} a t^{2}-L_{\mathrm{w} 14}-x_{0}\right)^{2} \\
& +\frac{40}{37} h\left(V_{0} t+\frac{1}{2} a t^{2}-L_{\mathrm{w} 14}-x_{0}\right),
\end{aligned}
$$

where $L_{\mathrm{w} 12}, L_{\mathrm{w} 13}$, and $L_{\mathrm{w} 14}$ denote the horizontal distances from wheel 1 centre to centres of wheel 2 , wheel 3 , and wheel 4 , respectively.
The vertical and pitch motions of the tank can be calculated accordingly. Different $h=0.2 \mathrm{~m}, 0.0 \mathrm{~m}$, and $-0.2 \mathrm{~m}$ representing a road bump, flat surface, and a pit are considered as the source of three different pitching excitations. The length of the tank $L$ and radius $R$ are taken to be $14 \mathrm{~m}$ and $1.125 \mathrm{~m}$ [32]. Table 3 lists the properties of the vehicle components. Note that these values are identical with those presented in [32]. The liquid tank is half-filled with water, i.e., $h_{1}=0.5 R$. The fundamental sloshing mode is considered in this case. The corresponding hydrodynamic coefficients are listed in Table 4 . The initial vehicle velocity $V_{0}$ is $10.0 \mathrm{~m} / \mathrm{s}$, and a constant deceleration due to braking is set to $a=-0.98 \mathrm{~m} / \mathrm{s}^{2}$ [22]. The deceleration of the vehicle with a rise and a decrease at the start and end of the vehicle braking is shown in Figure 11.

Figure 12 shows the longitudinal liquid sloshing forces and moments inside the tank during the course of vehicle braking for the case when $x_{0}=10 \mathrm{~m}$. In this case, the four wheels encounter the road bump or pit at $t=1.018 \mathrm{~s}, 1.58 \mathrm{~s}$, $2.79 \mathrm{~s}$, and $2.96 \mathrm{~s}$, respectively. In all cases, the vehicle was put to a halt at $t=11.20 \mathrm{~s}$, when the longitudinal acceleration $a$ drops to zero. This leads to a sharp change of the liquid sloshing force as shown in Figure 12. Such a sharp change may be smoothened by employing a more realistic model that accounts for the longitudinal contact between the wheels and the road. For the case of $h=0$ where no road bump or pit exists, the liquid sloshing is mainly induced by the sudden application of vehicle braking. The liquid sloshes at a long period due to the fact that the tank is quite long. It is also observed that the existence of a road bump or pit has a significant effect on the liquid sloshing force by inducing oscillations at a high frequency corresponding to the scenario of vehicle wheels encountering the road bump or pit. Such effect may be explained as due to the coupling between the longitudinal and rotational sloshing, which can be observed in the modal equation (see equation (9)). The maximum sloshing forces during vehicle brake are $25.1 \mathrm{kN}$, $21.2 \mathrm{kN}$, and $26.6 \mathrm{kN}$, respectively, for $h=-0.2 \mathrm{~m}, 0 \mathrm{~m}$, and $0.2 \mathrm{~m}$. However, the liquid sloshing moment is found to be governed by longitudinal deceleration of the vehicle while the presence of a road bump or pit has little effect on the sloshing moment. This is due to the fact that the liquid tank 
Table 3: Parameters of the vehicle model.

\begin{tabular}{|c|c|c|c|c|c|}
\hline Parameter & Unit & Value & Parameter & Unit & Value \\
\hline$M_{\mathrm{t}}$ & $\mathrm{kg}$ & 4819 & $l_{\mathrm{w} 1}$ & $\mathrm{~m}$ & 2.3 \\
\hline$M_{\mathrm{s}}$ & $\mathrm{kg}$ & 6400 & $l_{\mathrm{w} 2}$ & $\mathrm{~m}$ & 2.9 \\
\hline$I_{\mathrm{t}}$ & $\mathrm{kgm}^{2}$ & 10220 & $l_{\mathrm{w} 3}$ & $\mathrm{~m}$ & 2.206 \\
\hline$I_{\mathrm{s}}$ & $\mathrm{kgm}^{2}$ & 36854 & $l_{\mathrm{w} 4}$ & $\mathrm{~m}$ & 3.516 \\
\hline$k_{\mathrm{w}}$ & $\mathrm{N} / \mathrm{m}$ & 500000 & $l_{\mathrm{bt}}$ & $\mathrm{m}$ & 2.3 \\
\hline$k_{\mathrm{bt}}$ & $\mathrm{N} / \mathrm{m}$ & 100000 & $l_{\mathrm{bs}}$ & $\mathrm{m}$ & 8.494 \\
\hline$c_{\mathrm{w}}$ & $\mathrm{Ns} / \mathrm{m}$ & 10000 & $l_{\mathrm{w} 12}$ & $\mathrm{~m}$ & 5.2 \\
\hline$c_{\mathrm{bt}}$ & $\mathrm{Ns} / \mathrm{m}$ & 1000 & $l_{\mathrm{w} 13}$ & $\mathrm{~m}$ & 12.3 \\
\hline$l_{1}$ & $\mathrm{~m}$ & 2 & $l_{\mathrm{w} 14}$ & $\mathrm{~m}$ & 13.61 \\
\hline
\end{tabular}

TABle 4: Hydrodynamic coefficients.

\begin{tabular}{lcc}
\hline Parameter & Unit & $h_{l}=0.5 R$ \\
\hline$M_{1}$ & $\mathrm{~kg}$ & 27832.55 \\
$\omega_{(1,0)}$ & $\mathrm{rad} / \mathrm{s}$ & 0.675 \\
$\lambda_{1(1,0)}$ & $\mathrm{kg}$ & -85382.9 \\
$\lambda_{5(1,0)}$ & $\mathrm{kgm}$ & 82010 \\
$\mu_{(1,0)}$ & $\mathrm{kg}$ & 300440 \\
\hline
\end{tabular}

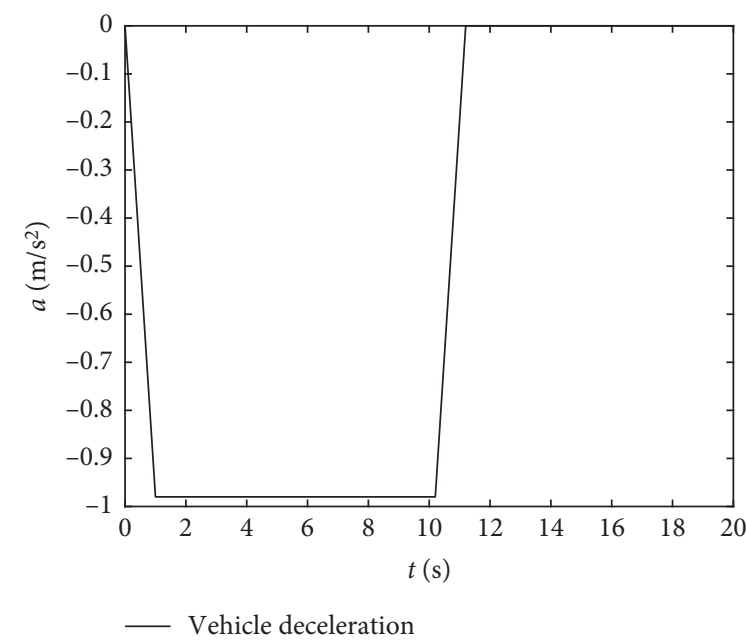

FIGURE 11: Deceleration during vehicle braking.

is quite long and the duration of excitation arising from the road bump or pit is rather short.

Figure 13 shows the effect of liquid sloshing on the dynamic pitch motion of the liquid tank during vehicle braking with the existence of a road bump. For comparison, the dynamic pitch motion of the same road tanker carrying solid cargo of equivalent mass and filling level is also plotted in Figure 13. It can be seen that the sloshing of liquid cargo significantly amplifies the pitch motion of the tank and thus reduces the stability of the vehicle. This agrees with the finding of another study presented in [33]. It is also found that the mean pitch motion of the liquid tank is larger than the solid cargo tank. This is reasonable in view that the inertia effect of liquid cargo during vehicle braking tends to impose a positive-pitch moment on the semitrailer.

The effect of different vehicle accelerations accounting for the presence of a road bump or pit is next studied. In

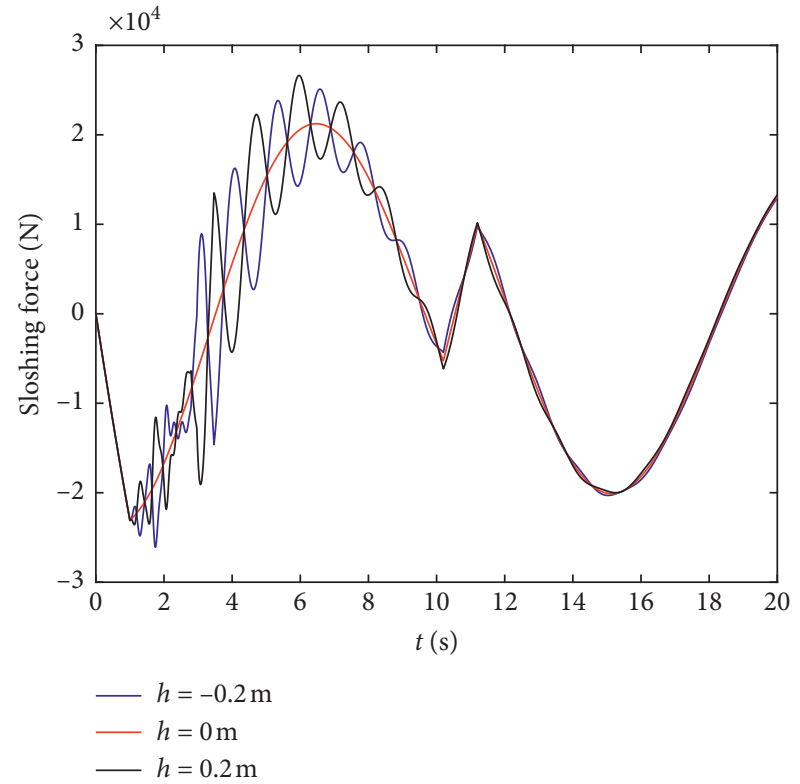

(a)

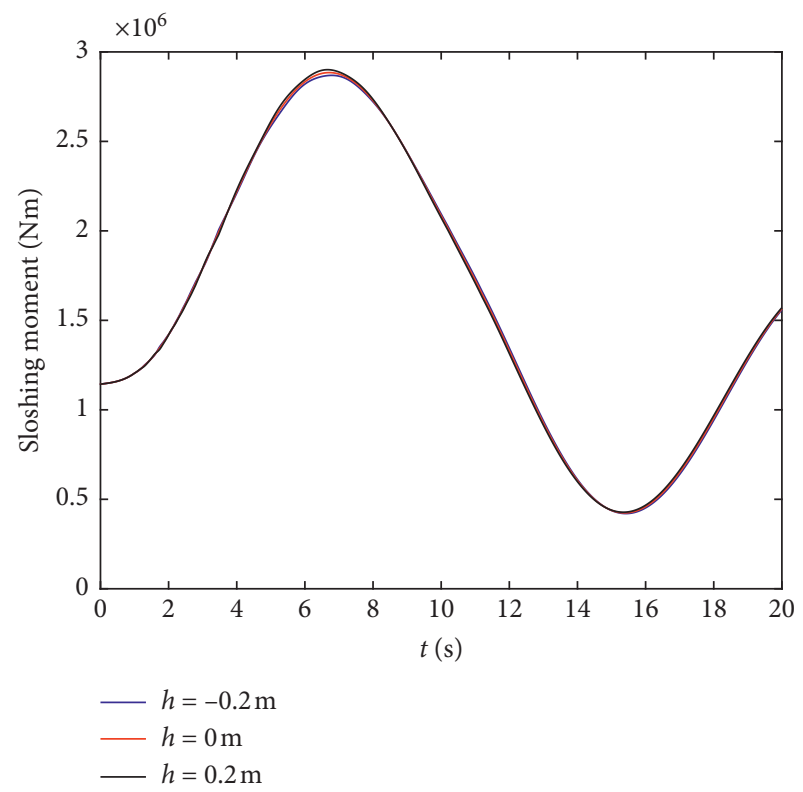

(b)

FIGURE 12: Road bump effect on liquid sloshing during vehicle braking: (a) sloshing force; (b) sloshing moment. 


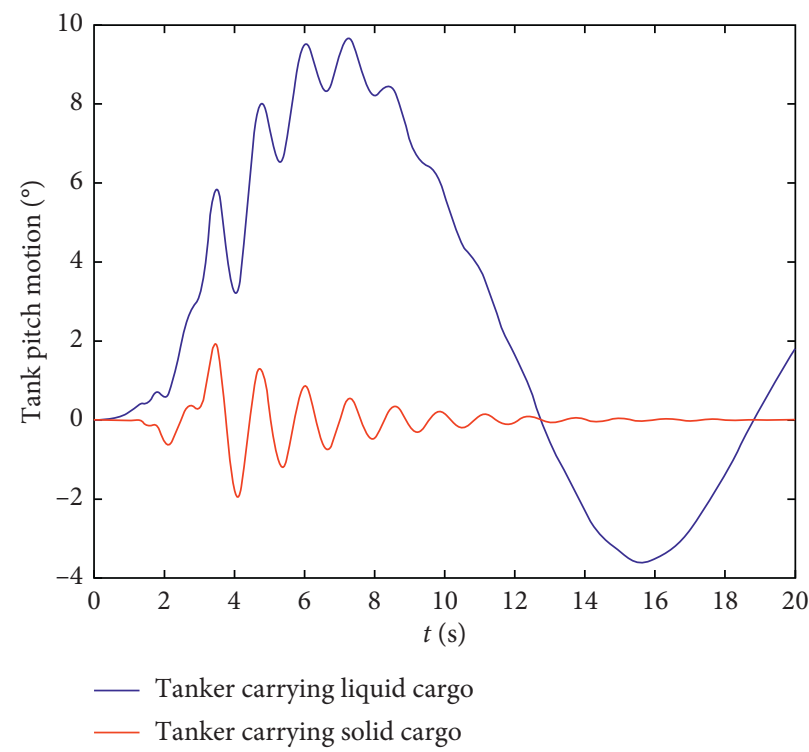

Figure 13: Effect of liquid sloshing on tank pitch motion.

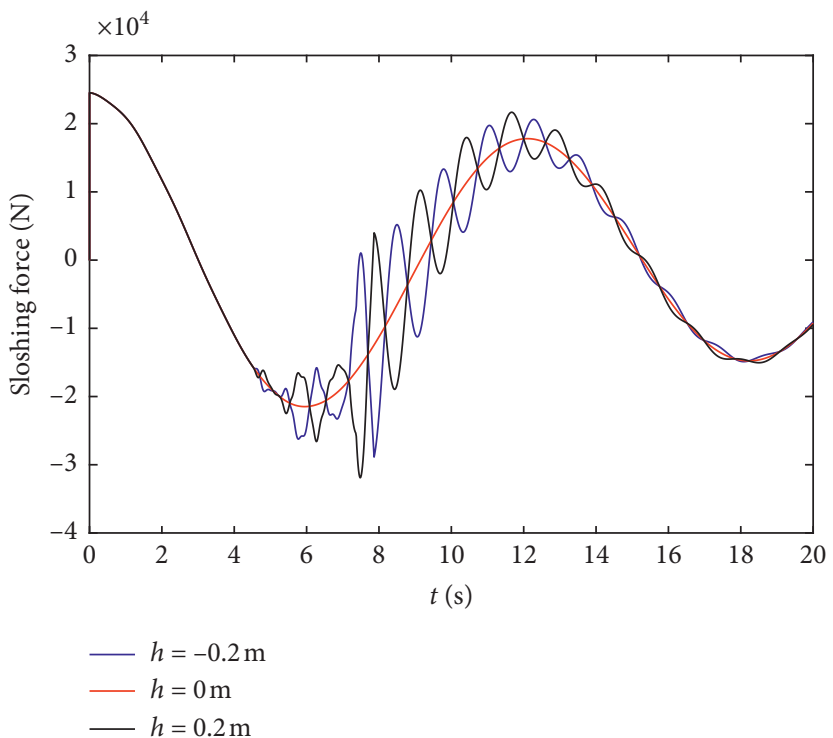

(a)

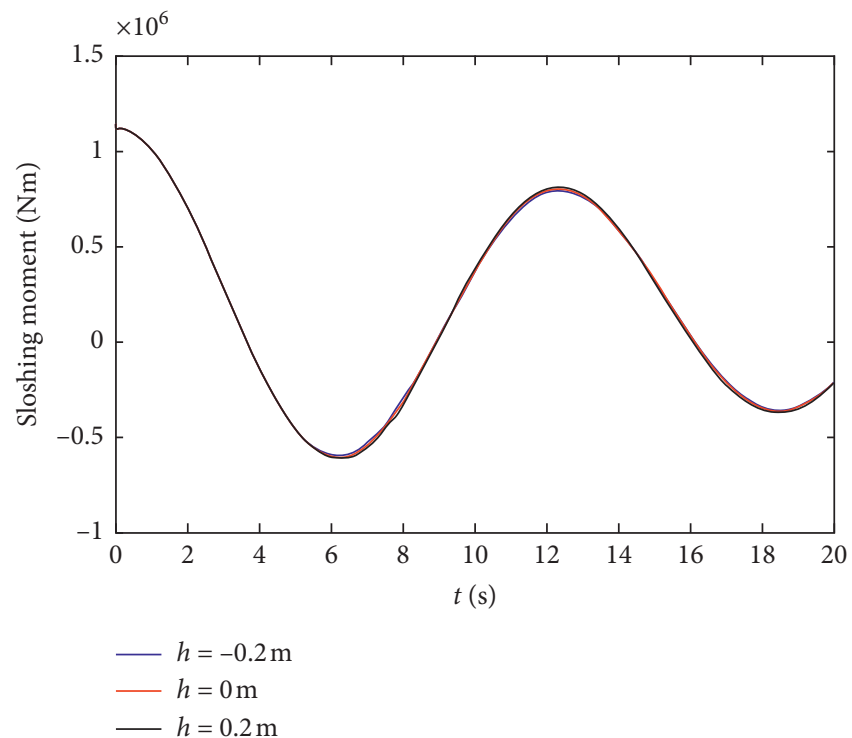

(b)

FIGURE 14: Sloshing force and moment during vehicle acceleration for $a=0.1 \mathrm{~g}$.

this case, the initial velocity $V_{0}$ is set to zero, while the initial distance $x_{0}$ and the bump shape remain unchanged. The sloshing forces and moments in a half-filled road tanker considering $h=-0.2 \mathrm{~m}, 0.0 \mathrm{~m}$, and $0.2 \mathrm{~m}$, subjected to a constant vehicle acceleration $a=0.1 \mathrm{~g}$ and $0.2 \mathrm{~g}$ are shown in Figures 14 and 15, respectively. When $a=0.1 \mathrm{~g}$, the four wheels encounter the road bump or pit at $t=4.52 \mathrm{~s}$, $5.55 \mathrm{~s}, 7.19 \mathrm{~s}$, and $7.37 \mathrm{~s}$, respectively. These time points are $t=3.19 \mathrm{~s}, 3.93 \mathrm{~s}, 5.08 \mathrm{~s}$, and $5.21 \mathrm{~s}$, respectively, for $a=0.2 \mathrm{~g}$. In all cases, the presence of a road bump or pit is found to cause significant fluctuations in the longitudinal sloshing force. However, the sloshing moment is almost unaffected, which agrees with aforementioned observations on vehicle deceleration. By comparing Figures 14 and 15, one may find that the degree of acceleration strongly affects the magnitude of the sloshing force and moment. The effect of road bump or pit on the sloshing force reduces as $a$ increases due to the fact that the entire road tanker travels across the road bump/pit in a shorter duration at a higher encounter speed. 


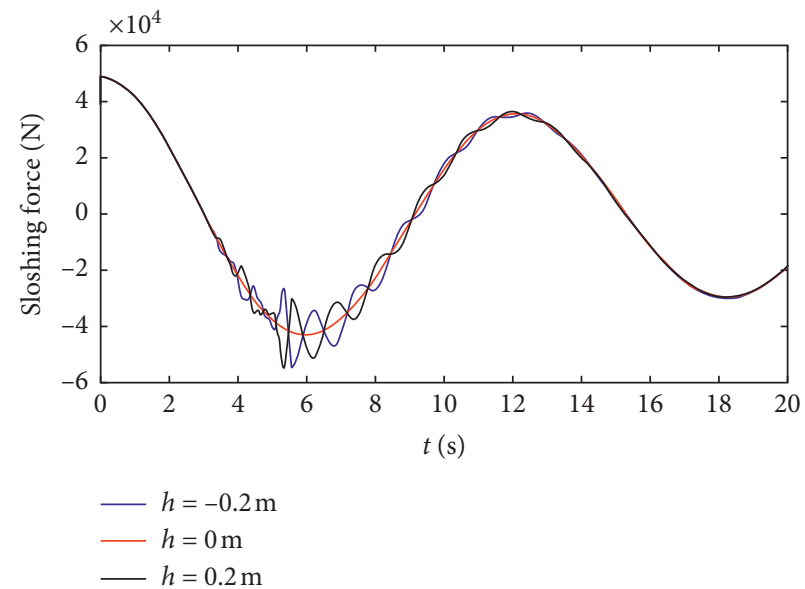

(a)

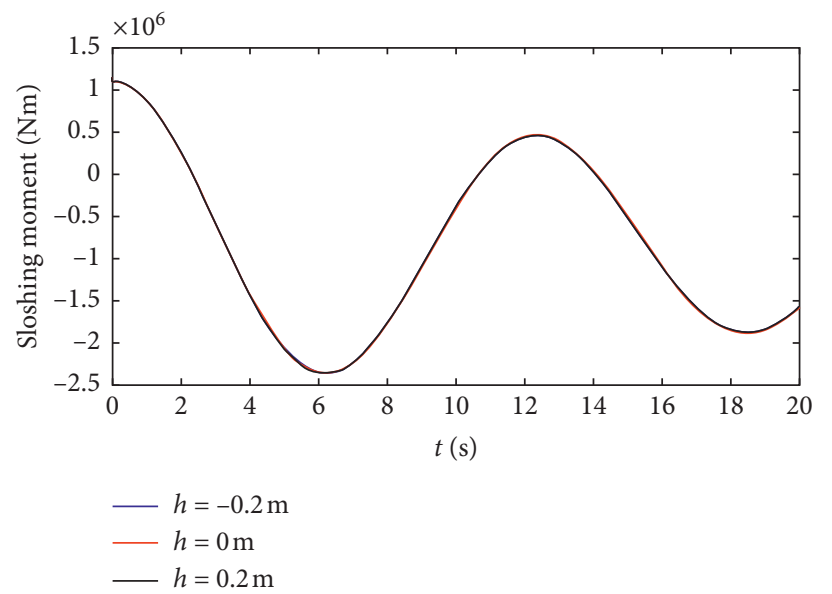

(b)

FIGURE 15: Sloshing force and moment during vehicle acceleration for $a=0.2 g$.

\section{Conclusions}

This paper presents a novel approach for determining the transient liquid sloshing in road tankers subjected to complex vehicle motions in the time domain. Linear modal equations based on the linear potential flow theory are employed, and the time-independent hydrodynamic coefficients required to establish the motion equation are derived from the added mass obtained by using available frequency-domain boundary element software.

With an assumed free surface shape function, the hydrodynamic coefficients are conveniently calculated, and the modal equations describing 3 -dimensional sloshing problem can be fully coupled with the mechanical vehicle model. The accuracy of the proposed approach is examined in detail by comparison with available numerical and experimental results in the literature. Satisfactory agreement between the results is obtained for different case studies. However, the assumptions of linear potential flow theory restrict the application of this approach to mild sloshing, which requires the external excitation frequency to be away from the natural frequency of the liquid and the excitation amplitude to be small.

A parametric study was carried out to allow for the effect of a coupled vehicle translational and rotational motion on the liquid sloshing behaviour. The results show that the vehicle pitch motion has a significant effect on the sloshing force, which should not be neglected in practical applications where road surface roughness and/or road bump/pit exist. Parametric study has shown that the amplitude of sloshing force is jointly determined by the surface roughness, vehicle properties, acceleration/deceleration of vehicle, and hydrodynamic properties of the liquid cargo.

Although this study focuses on the sloshing inside a road tanker subject to a selected combinations of vehicle motions, the method described here applies to a $3 \mathrm{D}$ vehicle accounting for complicated scenario of all 6-degree-of-freedom motions as well. Furthermore, the accuracy of the proposed model can be further improved by including more sloshing modes in the computation. Compared with computational intensive CFD simulations, this approach has the advantage of computational efficiency for solving linear sloshing problems.

\section{Data Availability}

The numerical data used to support the findings of this study are available from the corresponding author upon request.

\section{Conflicts of Interest}

The authors declare that they have no conflicts of interest.

\section{References}

[1] R. A. Ibrahim, V. N. Pilipchuk, and T. Ikeda, "Recent advances in liquid sloshing dynamics," Applied Mechanics Reviews, vol. 54, no. 2, pp. 133-199, 2001.

[2] B. Serván-Camas, J. L. Cercós-Pita, J. Colom-Cobb, J. GarcíaEspinosa, and A. Souto-Iglesias, "Time domain simulation of coupled sloshing-seakeeping problems by SPH-FEM coupling," Ocean Engineering, vol. 123, pp. 383-396, 2016.

[3] Y.-L. Li, R.-C. Zhu, G.-P. Miao, and J. Fan, "Simulation of tank sloshing based on OpenFOAM and coupling with ship motions in time domain," Journal of Hydrodynamics, vol. 24, no. 3, pp. 450-457, 2012.

[4] J. R. Saripilli and D. Sen, "Numerical studies on effects of slosh coupling on ship motions and derived slosh loads," Applied Ocean Research, vol. 76, pp. 71-87, 2018.

[5] J. Romero Navarrete and F. Otremba, "Experimental and theoretical modeling of cargo sloshing during braking," in Proceedings of the ASME 2016 International Mechanical Engineering Congress and Exposition, Phoenix, AZ, USA, November 2016.

[6] A. Kolaei, S. Rakheja, and M. J. Richard, "Effects of tank crosssection on dynamic fluid slosh loads and roll stability of a partly-filled tank truck," European Journal of Mechanics-B/ Fluids, vol. 46, pp. 46-58, 2014.

[7] K. Modaressi-Tehrani, S. Rakheja, and I. Stiharu, "Three-dimensional analysis of transient slosh within a partly-filled 
tank equipped with baffles," Vehicle System Dynamics, vol. 45, no. 6, pp. 525-548, 2007.

[8] A. Kolaei, S. Rakheja, and M. J. Richard, "Coupled multimodal fluid-vehicle model for analysis of anti-slosh effectiveness of longitudinal baffles in a partially-filled tank vehicle," Journal of Fluids and Structures, vol. 70, pp. 519-536, 2017.

[9] S. M. Hasheminejad, M. M. Mohammadi, and M. Jarrahi, "Liquid sloshing in partly-filled laterally-excited circular tanks equipped with baffles," Journal of Fluids and Structures, vol. 44, pp. 97-114, 2014.

[10] W. Wang, Y. Peng, Q. Zhang, L. Ren, and Y. Jiang, "Sloshing of liquid in partially liquid filled toroidal tank with various baffles under lateral excitation," Ocean Engineering, vol. 146, pp. 434-456, 2017.

[11] B. Nicolsen, L. Wang, and A. Shabana, "Nonlinear finite element analysis of liquid sloshing in complex vehicle motion scenarios," Journal of Sound and Vibration, vol. 405, pp. 208-233, 2017.

[12] Y. Zhuang and D. Wan, "Numerical study on ship motion fully coupled with LNG tank sloshing in CFD method," International Journal of Computational Methods, vol. 16, no. 6, Article ID 1840022, 2017.

[13] J. L. Cercos-Pita, G. Bulian, L. Pérez-Rojas, and A. Francescutto, "Coupled simulation of nonlinear ship motions and a free surface tank," Ocean Engineering, vol. 120, pp. 281-288, 2016.

[14] G. Bulian and J. L. Cercos-Pita, "Co-simulation of ship motions and sloshing in tanks," Ocean Engineering, vol. 152, pp. 353-376, 2018.

[15] M. J. Ketabdari and H. Saghi, "Parametric study for optimization of storage tanks considering sloshing phenomenon using coupled BEM-FEM," Applied Mathematics and Computation, vol. 224, pp. 123-139, 2013.

[16] O. M. Faltinsen and A. N. Timokha, Sloshing, Cambridge University Press, Cambridge, UK, 2009.

[17] E. di Gialleonardo, A. Premoli, S. Gallazzi, and S. Bruni, "Sloshing effects and running safety in railway freight vehicles," Vehicle System Dynamics, vol. 51, no. 10, pp. 1640-1654, 2013.

[18] M. Farid, N. Levy, and O. V. Gendelman, "Vibration mitigation in partially liquid-filled vessel using passive energy absorbers," Journal of Sound and Vibration, vol. 406, pp. 51-73, 2017.

[19] M. Farid and O. V. Gendelman, "Internal resonances and dynamic responses in equivalent mechanical model of partially liquid-filled vessel," Journal of Sound and Vibration, vol. 379, pp. 191-212, 2016.

[20] T. L. Manderbacka, V. Jacob, T. Carriot, T. Mikkola, and J. E. Matusiak, "Sloshing forces on a tank with two compartments, application of the pendulum model and CFD," in Proceedings of the International Conference on Offshore Mechanics and Arctic Engineering-OMAE, San Francisco, CA, USA, June 2014.

[21] G. I. Bogomaz, O. M. Markova, and Y. G. Chernomashentseva, "Mathematical modelling of vibrations and loading of railway tanks taking into account the liquid cargo mobility," Vehicle System Dynamics, vol. 30, no. 3-4, pp. 285-294, 1998.

[22] A. Kolaei, S. Rakheja, and M. J. Richard, "Three-dimensional dynamic liquid slosh in partially-filled horizontal tanks subject to simultaneous longitudinal and lateral excitations," European Journal of Mechanics-B/Fluids, vol. 53, pp. 251-263, 2015.

[23] S. M. Hasheminejad and M. Aghabeigi, "Transient sloshing in half-full horizontal elliptical tanks under lateral excitation,"
Journal of Sound and Vibration, vol. 330, no. 14, pp. 35073525, 2011.

[24] Bureau Veritas, HydroStar for Experts User Manual, Bureau Veritas, Neuilly-Sur-Seine, France, 2018.

[25] S. Malenica, M. Zalar, and X. B. Chen, "Dynamic coupling of seakeeping and sloshing," in Proceedings of the International Offshore and Polar Engineering Conference, Honolulu, HI, USA, May 2003.

[26] Wamit Inc., WAMIT User Manual, 2018, http://www.wamit. com/manualupdate/v72_manual.pdf.

[27] J. N. Newman, "Wave effects on vessels with internal tanks," in Proceedings of the 20th Workshop on Water Waves and Floating Bodies, Longyearbyen, Norway, May 2005.

[28] X. Chen, "Hydrodynamics in offshore and naval applicationspart I, keynote lecture," in Proceedings of the 6th International Conference on Hydrodynamics, Perth, Australia, November 2004.

[29] C. C. Mei, "The applied dynamics of ocean surface waves," in Advanced Series on Ocean Engineering, World Scientific, Singapore, 1989.

[30] X. Chen, R.-C. Zhu, W.-J. Zhou, and J. Zhao, "A 3D multidomain high order boundary element method to evaluate time domain motions and added resistance of ship in waves," Ocean Engineering, vol. 159, pp. 112-128, 2018.

[31] H. Akyildiz and E. Ünal, "Experimental investigation of pressure distribution on a rectangular tank due to the liquid sloshing," Ocean Engineering, vol. 32, no. 11-12, pp. 15031516, 2005.

[32] M. M. Jalili, M. Motavasselolhagh, R. Fatehi, and M. Sefid, "Investigation of sloshing effects on dynamic response of an articulated vehicle carrying liquids," Proceedings of the Institution of Mechanical Engineers, Part D: Journal of Automobile Engineering, vol. 232, no. 10, pp. 1385-1401, 2018.

[33] J. Dai, M. Han, and K. K. Ang, "Moving element analysis of partially filled freight trains subject to abrupt braking," International Journal of Mechanical Sciences, vol. 151, pp. 85-94, 2019. 


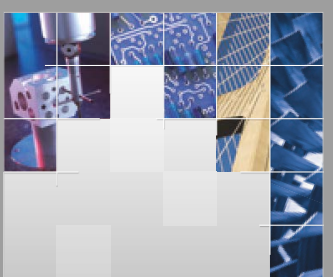

\section{Enfincering}
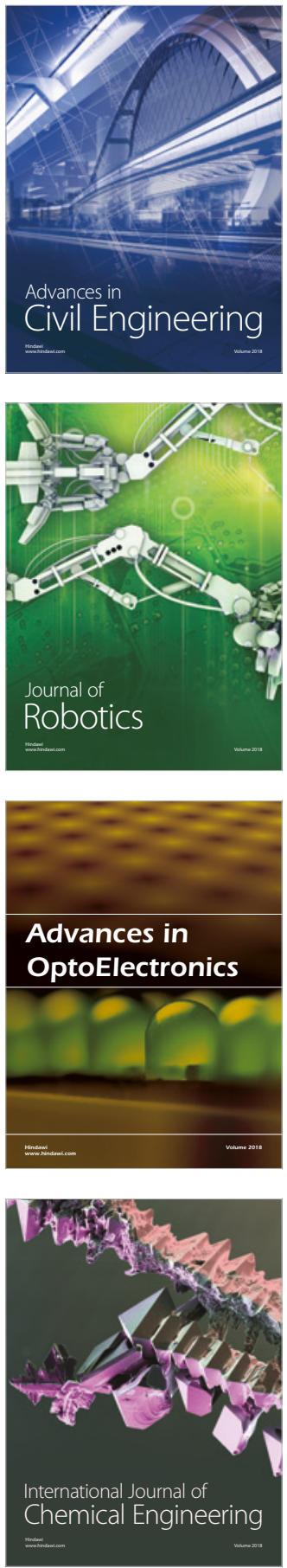

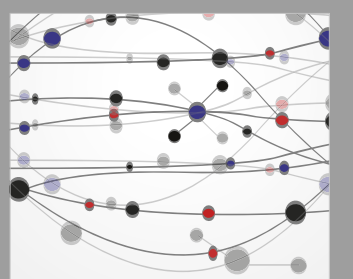

\section{Rotating \\ Machinery}

The Scientific World Journal

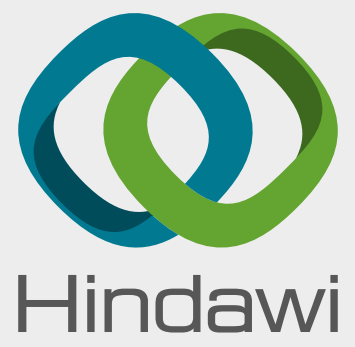

Submit your manuscripts at

www.hindawi.com
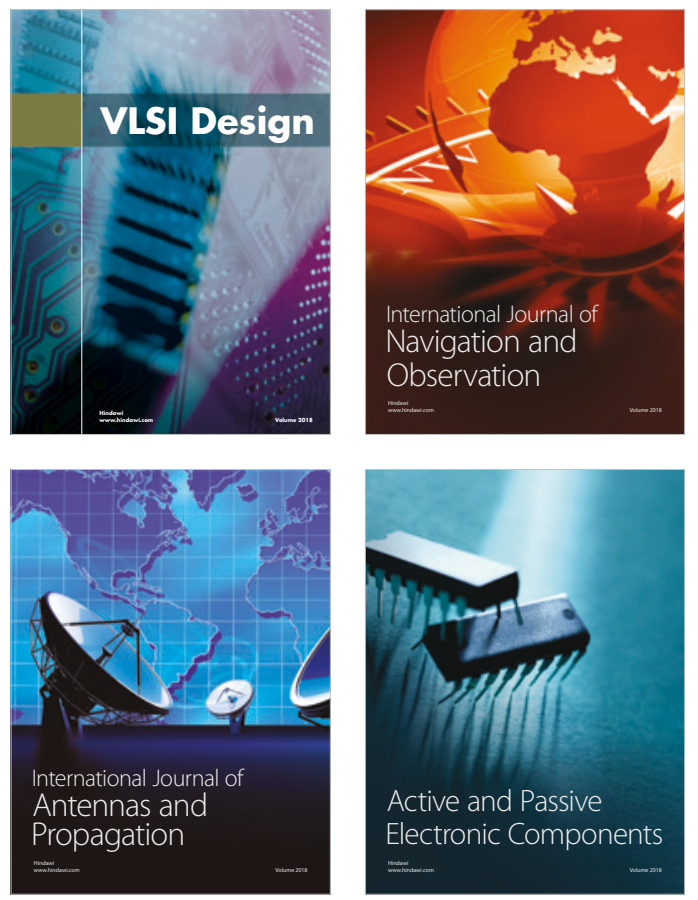
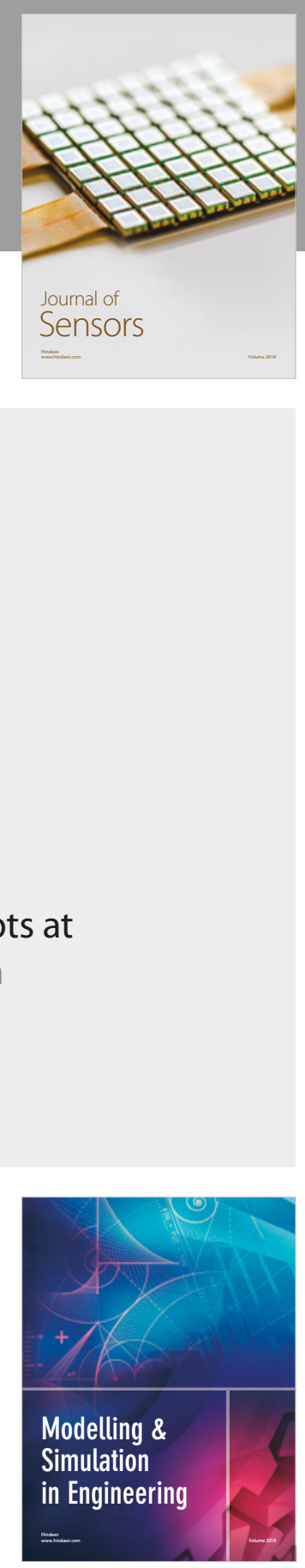

\section{Advances \\ Multimedia}
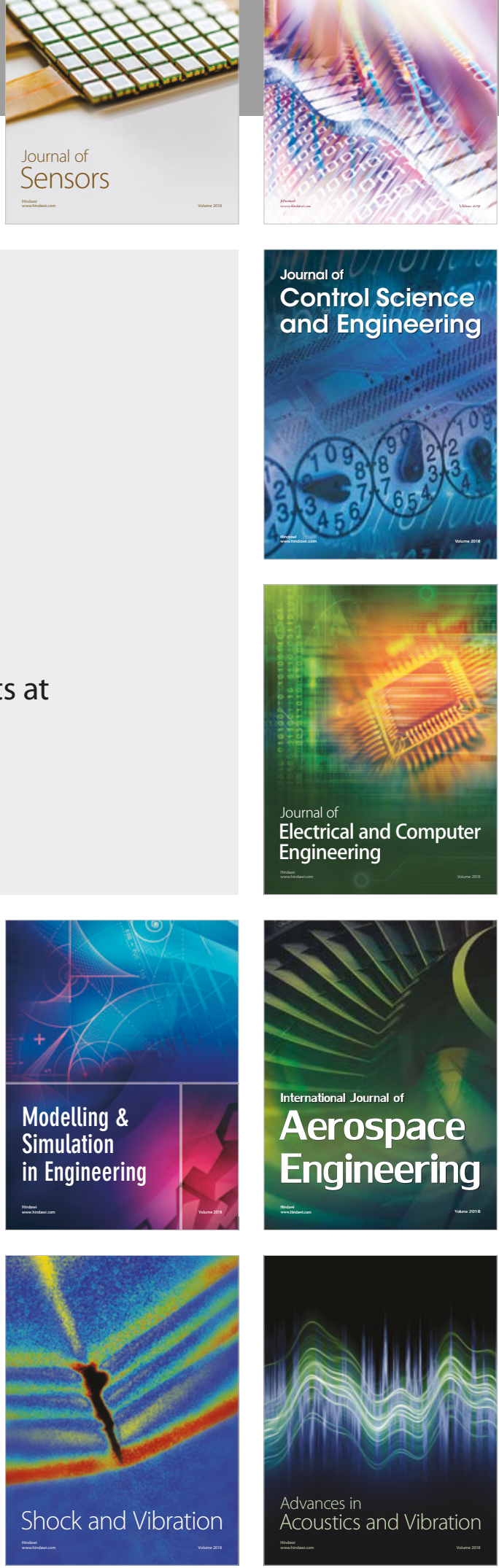